\title{
Combined Reduced-Rank Transform
}

\author{
Anatoli TOROKHTI and Phil HOWLETT
}

School of Mathematics and Statistics, University of South Australia, Australia

E-mail: anatoli.torokhti@unisa.edu.au,phil.howlett@unisa.edu.au

URL: http://people.unisa.edu.au/Anatoli.Torokhti

http://people.unisa.edu.au/Phil.Howlett

Received November 25, 2005, in final form March 22, 2006; Published online April 07, 2006

Original article is available at http://www.emis.de/journals/SIGMA/2006/Paper039/

\begin{abstract}
We propose and justify a new approach to constructing optimal nonlinear transforms of random vectors. We show that the proposed transform improves such characteristics of rank-reduced transforms as compression ratio, accuracy of decompression and reduces required computational work. The proposed transform $\mathcal{T}_{p}$ is presented in the form of a sum with $p$ terms where each term is interpreted as a particular rank-reduced transform. Moreover, terms in $\mathcal{T}_{p}$ are represented as a combination of three operations $\mathcal{F}_{k}, \mathcal{Q}_{k}$ and $\boldsymbol{\varphi}_{k}$ with $k=1, \ldots, p$. The prime idea is to determine $\mathcal{F}_{k}$ separately, for each $k=1, \ldots, p$, from an associated rank-constrained minimization problem similar to that used in the Karhunen-Loève transform. The operations $\mathcal{Q}_{k}$ and $\varphi_{k}$ are auxiliary for finding $\mathcal{F}_{k}$. The contribution of each term in $\mathcal{T}_{p}$ improves the entire transform performance. A corresponding unconstrained nonlinear optimal transform is also considered. Such a transform is important in its own right because it is treated as an optimal filter without signal compression. A rigorous analysis of errors associated with the proposed transforms is given.
\end{abstract}

Key words: best approximation; Fourier series in Hilbert space; matrix computation

2000 Mathematics Subject Classification: 41A29

\section{Introduction}

Methods of data dimensionality reduction $[1,2,3,4,5,6,7,8,9,10,11,12,13,14,15,16,17,18$, 19, 20, 21, 22, 23, 24] have been applied successfully to many applied problems. The diversity of applications has stimulated a considerable increase in the study of data dimensionality reduction in recent decades. Significant recent results in this challenging research area are described, in particular, in references $[4,5,6,7,8,9,10,11,12,13,14,15,16,17,18,19,20,21,22,23,24]$. The known methods concern both a probabilistic setting (as in $[5,6,7,8,9,10,16,17,18$, $19,20,21,22,23,24,25,26,27,28]$ ) and deterministic setting (as in $[12,13,14,15]$ ) in the dimensionality reduction. The associated techniques are often based on the use of reduced-rank operators.

In this paper, a further advance in the development of reduced-rank transforms is presented. We study a new approach to data dimensionality reduction in a probabilistic setting based on the development of ideas presented in [5, 6, 7, 26, 27, 28, 29].

Motivation for the proposed approach arises from the following observation. In general, the reduced-rank transform consists of the three companion operations which are filtering, compression and reconstruction $[5,6,7,16,26]$. Filtering and compression are performed simultaneously to estimate a reference signal $\boldsymbol{x}$ with $m$ components from noisy observable data $\boldsymbol{y}$ and to filter and reduce the data to a shorter vector $\hat{\boldsymbol{x}}$ with $\eta$ components, $\eta<m$. Components of $\hat{\boldsymbol{x}}$ are often called principal components [4]. The quotient $\eta / m$ is called the compression ratio. Reconstruction returns a vector $\tilde{\boldsymbol{x}}$ with $m$ components so that $\tilde{\boldsymbol{x}}$ should be close to the original $\boldsymbol{x}$. 
It is natural to perform these three operations so that the reconstruction error and the related computational burden are minimal.

As a result, the performance of the reduced-rank transform is characterized by three issues which are (i) associated accuracy, (ii) compression ratio, and (iii) computational work.

For a given compression ratio, the Karhunen-Loève transform (KLT) [5, 6, 7] minimizes the reconstruction error over the class of all linear reduced-rank transforms. Nevertheless, it may happen that the accuracy and compression ratio associated with the KLT are still not satisfactory. In such a case, an improvement in the accuracy and compression ratio can be achieved by a transform with a more general structure than that of the KLT. Special non-linear transforms have been studied in $[16,17,18,19,20,21,22,23,24,25,26,27,28,29,30,31,32,33,34]$ using transform structures developed from the generalised Volterra polynomials. Nevertheless, the transforms $[16,26,27,28,29]$ imply a substantial computational burden associated with the large number $N$ of terms required by the underlying Volterra polynomial structure.

Our objective is to justify a new transform that may have both accuracy and compression ratio better than those of the known transforms [5, 6, 7, 26, 27, 28, 29]. A related objective is to find a way to reduce the associated computational work compared with that implied by the transforms [26, 27, 28, 29]. The analysis of these issues is given in Sections 4, 5.2.2 (Remark 4), 5.2.3 and 5.2.4.

In Section 5.2.5, we show that the proposed approach generalizes the Fourier series in Hilbert space, the Wiener filter, the Karhunen-Loève transform and the transforms given in [26, 27, 29].

\section{Method description}

We use the following notation:

$(\Omega, \Sigma, \mu)$ is a probability space, where $\Omega=\{\omega\}$ is the set of outcomes, $\Sigma$ a $\sigma$-field of measurable subsets of $\Omega$ and $\mu: \Sigma \rightarrow[0,1]$ an associated probability measure on $\Sigma$ with $\mu(\Omega)=1$; $\boldsymbol{x} \in L^{2}\left(\Omega, \mathbb{R}^{m}\right)$ and $\boldsymbol{y} \in L^{2}\left(\Omega, \mathbb{R}^{n}\right)$ are random vectors with realizations $x=\boldsymbol{x}(\omega) \in \mathbb{R}^{m}$ and $y=\boldsymbol{y}(\omega) \in \mathbb{R}^{n}$, respectively.

Each matrix $M \in \mathbb{R}^{m \times n}$ defines a bounded linear transformation $\mathcal{M}: L^{2}\left(\Omega, \mathbb{R}^{n}\right) \rightarrow L^{2}\left(\Omega, \mathbb{R}^{m}\right)$ via the formula $[\mathcal{M} \boldsymbol{y}](\omega)=M \boldsymbol{y}(\omega)$ for each $\omega \in \Omega$. We note that there are many bounded linear transformations from $L^{2}\left(\Omega, \mathbb{R}^{n}\right)$ into $L^{2}\left(\Omega, \mathbb{R}^{m}\right)$ that cannot be written in the form $[\mathcal{M} \boldsymbol{y}](\omega)=M \boldsymbol{y}(\omega)$ for each $\omega \in \Omega$. A trivial example is $\mathcal{A}: L^{2}\left(\Omega, \mathbb{R}^{n}\right) \rightarrow L^{2}\left(\Omega, \mathbb{R}^{m}\right)$ given by $\mathcal{A}(\boldsymbol{y})=\int_{\Omega} \boldsymbol{y}(\omega) d \mu(\omega)$.

Throughout the paper, the calligraphic character letters denote operators defined similarly to $\mathcal{M}$.

Let $\boldsymbol{g}=\left[\boldsymbol{g}_{1} \ldots \boldsymbol{g}_{m}\right]^{T} \in L^{2}\left(\Omega, \mathbb{R}^{m}\right)$ and $\boldsymbol{h}=\left[\boldsymbol{h}_{1} \ldots \boldsymbol{h}_{n}\right]^{T} \in L^{2}\left(\Omega, \mathbb{R}^{n}\right)$ be random vectors with $\boldsymbol{g}_{i}, \boldsymbol{h}_{k} \in L^{2}(\Omega, \mathbb{R})$ for $i=1, \ldots, m, k=1, \ldots, n$. For all $i=1, \ldots, m$ and $k=1, \ldots, n$, we set

$$
\begin{aligned}
& E\left[\boldsymbol{g}_{i}\right]=\int_{\Omega} \boldsymbol{g}_{i}(\omega) d \mu(\omega), \quad E\left[\boldsymbol{g}_{i} \boldsymbol{h}_{k}\right]=\int_{\Omega} \boldsymbol{g}_{i}(\omega) \boldsymbol{h}_{k}(\omega) d \mu(\omega), \\
& E_{g h}=E\left[\boldsymbol{g} \boldsymbol{h}^{T}\right]=\left\{E\left[\boldsymbol{g}_{i} \boldsymbol{h}_{k}\right]\right\} \in \mathbb{R}^{m \times n} \quad \text { and } \quad E_{g}=E[\boldsymbol{g}]=\left\{E\left[\boldsymbol{g}_{i}\right]\right\} \in \mathbb{R}^{m} .
\end{aligned}
$$

We also write

$$
\mathbb{E}_{g h}=E\left[\left(\boldsymbol{g}-E_{g}\right)\left(\boldsymbol{h}-E_{h}\right)^{T}\right]=E_{g h}-E[\boldsymbol{g}] E\left[\boldsymbol{h}^{T}\right] .
$$

Achievement of the above objectives is based on the presentation of the proposed transform in the form of a sum with $p$ terms (3) where each term is interpreted as a particular rank-reduced transform. Moreover, terms in (3) are represented as a combination of three operations $\mathcal{F}_{k}$, $\mathcal{Q}_{k}$ and $\boldsymbol{\varphi}_{k}$ for each $k=1, \ldots, p$, where $\boldsymbol{\varphi}_{k}$ is nonlinear. The prime idea is to determine $\mathcal{F}_{k}$ 
separately, for each $k=1, \ldots, p$, from an associated rank-constrained minimization problem similar to that in the KLT. The operations $\mathcal{Q}_{k}$ and $\varphi_{k}$ are auxiliary for finding $\mathcal{F}_{k}$. It is natural to expect that a contribution of each term in (3) will improve the entire transform performance.

To realize such a scheme, we choose the $\mathcal{Q}_{k}$ as orthogonal/orthonormal operators (see Section 3). Then each $\mathcal{F}_{k}$ can be determined independently for each individual problem (33) or (56) below. Next, operators $\varphi_{k}$ are used to reduce the number of terms from $N$ (as in $[16,26,27,28,29])$ to $p$ with $p \ll N$. For example, this can be done when we choose $\varphi_{k}$ in the form presented in Section 5.2.4. Moreover, the composition of operators $\mathcal{Q}_{k}$ and $\varphi_{k}$ allows us to reduce the related covariance matrices to the identity matrix or to a block-diagonal form with small blocks. Remark 4 in Section 5.2.2 gives more details in this regard. The computational work associated with such blocks is much less than that for the large covariance matrices in $[16,26,27,28,29]$.

To regulate accuracy associated with the proposed transform and its compression ratio, we formulate the problem in the form (6)-(7) where (7) consists of $p$ constraints. It is shown in Remark 2 of Section 4, and in Sections 5.2.1, 5.2.2 and 5.2.4 that such a combination of constraints allows us to equip the proposed transforms with several degrees of freedom.

The structure of our transform is presented in Section 3 and the formal statement of the problem in Section 4. In Section 5, we determine operators $\mathcal{Q}_{k}$ and $\mathcal{F}_{k}$ (Lemmata 1 and 3, and Theorems 1 and 2, respectively).

\section{Structure of the proposed transform}

\subsection{Generic form}

The proposed transform $\mathcal{T}_{p}$ is presented in the form

$$
\mathcal{T}_{p}(\boldsymbol{y})=f+\sum_{k=1}^{p} \mathcal{F}_{k} \mathcal{Q}_{k} \boldsymbol{\varphi}_{k}(\boldsymbol{y})=f+\mathcal{F}_{1} \mathcal{Q}_{1} \boldsymbol{\varphi}_{1}(\boldsymbol{y})+\cdots+\mathcal{F}_{p} \mathcal{Q}_{p} \boldsymbol{\varphi}_{p}(\boldsymbol{y})
$$

where $f \in \mathbb{R}^{m}, \boldsymbol{\varphi}_{k}: L^{2}\left(\Omega, \mathbb{R}^{n}\right) \rightarrow L^{2}\left(\Omega, \mathbb{R}^{n}\right), \mathcal{Q}_{1}, \ldots, \mathcal{Q}_{p}: L^{2}\left(\Omega, \mathbb{R}^{n}\right) \rightarrow L^{2}\left(\Omega, \mathbb{R}^{n}\right)$ and $\mathcal{F}_{k}:$ $L^{2}\left(\Omega, \mathbb{R}^{n}\right) \rightarrow L^{2}\left(\Omega, \mathbb{R}^{m}\right)$.

In general, one can put $\boldsymbol{x} \in L^{2}\left(\Omega, H_{X}\right), \boldsymbol{y} \in L^{2}\left(\Omega, H_{Y}\right), \boldsymbol{\varphi}_{k}: L^{2}\left(\Omega, H_{Y}\right) \rightarrow L^{2}\left(\Omega, H_{k}\right)$, $\mathcal{Q}_{k}: L^{2}\left(\Omega, H_{k}\right) \rightarrow L^{2}\left(\Omega, \tilde{H}_{k}\right)$ and $\mathcal{F}_{k}: L^{2}\left(\Omega, \tilde{H}_{k}\right) \rightarrow L^{2}\left(\Omega, H_{X}\right)$ with $H_{X}, H_{Y}, H_{k}$ and $\tilde{H}_{k}$ separable Hilbert spaces, and $k=1, \ldots, p$.

In (3), the vector $f$ and operators $\mathcal{F}_{1}, \ldots, \mathcal{F}_{p}$ are determined from the minimization problem (6)-(7) given in the Section 4. Operators $\mathcal{Q}_{1}, \ldots, \mathcal{Q}_{p}$ in (3) are orthogonal (orthonormal) in the sense of the Definition 1 in Section 4 (in this regard, see also Remark 3 in Section 5.1).

To demonstrate and justify flexibility of the transform $\mathcal{T}_{p}$ with respect to the choice of $\boldsymbol{\varphi}_{1}, \ldots, \boldsymbol{\varphi}_{p}$ in (3), we mainly study the case where $\boldsymbol{\varphi}_{1}, \ldots, \boldsymbol{\varphi}_{p}$ are arbitrary. Specifications of

$\boldsymbol{\varphi}_{1}, \ldots, \boldsymbol{\varphi}_{p}$ are presented in Sections 3.2, 5.2.4 and 5.2.5 where we also discuss the benefits associated with some particular forms of $\boldsymbol{\varphi}_{1}, \ldots, \boldsymbol{\varphi}_{p}$.

\subsection{Some particular cases}

Particular cases of the model $\mathcal{T}_{p}$ are associated with specific choices of $\boldsymbol{\varphi}_{k}, \mathcal{Q}_{k}$ and $\mathcal{F}_{k}$. Some examples are given below.

(i) If $H_{X}=H_{Y}=\mathbb{R}^{n}$ and $H_{k}=\tilde{H}_{k}=\mathbb{R}^{n k}$ where $\mathbb{R}^{n k}$ is the $k$ th degree of $\mathbb{R}^{n}$, then (3) generalises the known transform structures [16, 26, 27, 28, 29]. The models [16, 26, 27, 28, 29] follow from (3) if $\boldsymbol{\varphi}_{k}(\boldsymbol{y})=\boldsymbol{y}^{k}$ where $\boldsymbol{y}^{k}=(\boldsymbol{y}, \ldots, \boldsymbol{y}) \in L^{2}\left(\Omega, \mathbb{R}^{n k}\right), \mathcal{Q}_{k}=\mathcal{I}$, where $\mathcal{I}$ is the identity operator, and if $\mathcal{F}_{k}$ is a $k$-linear operator. It has been shown in [16, 26, 27, 28, 29] that 
such a form of $\boldsymbol{\varphi}_{k}$ leads to a significant improvement in the associated accuracy. See Section 5.2.5 for more details.

(ii) If $\boldsymbol{\varphi}_{k}: L^{2}\left(\Omega, H_{Y}\right) \rightarrow L^{2}\left(\Omega, H_{X}\right)$ and $\left\{\boldsymbol{u}_{1}, \boldsymbol{u}_{2}, \ldots\right\}$ is a basis in $L^{2}\left(\Omega, H_{X}\right)$ then $\boldsymbol{\varphi}_{k}$ and $\mathcal{Q}_{k}$ can be chosen so that $\boldsymbol{\varphi}_{k}(\boldsymbol{y})=\boldsymbol{u}_{k}$ and $\mathcal{Q}_{k}=\mathcal{I}$, respectively. As a result, in this particular case, $\mathcal{T}_{p}(\boldsymbol{y})=f+\sum_{k=1}^{p} \mathcal{F}_{k}\left(\boldsymbol{u}_{k}\right)$.

(iii) A similar case follows if $\varphi_{k}: L^{2}\left(\Omega, H_{Y}\right) \rightarrow L^{2}\left(\Omega, H_{k}\right)$ is arbitrary but $\mathcal{Q}_{k}: L^{2}\left(\Omega, H_{k}\right) \rightarrow$ $L^{2}\left(\Omega, \tilde{H}_{k}\right)$ is defined so that $\mathcal{Q}_{k}\left[\boldsymbol{\varphi}_{k}(\boldsymbol{y})\right]=\boldsymbol{v}_{k}$ with $k=1, \ldots, p$ where $\left\{\boldsymbol{v}_{1}, \boldsymbol{v}_{2}, \ldots\right\}$ is a basis in $L^{2}\left(\Omega, \tilde{H}_{k}\right)$. Then $\mathcal{T}_{p}(\boldsymbol{y})=f+\sum_{k=1}^{p} \mathcal{F}_{k}\left(\boldsymbol{v}_{k}\right)$.

(iv) Let $\tilde{\boldsymbol{x}}^{(1)}, \ldots, \tilde{\boldsymbol{x}}^{(p)}$ be estimates of $\boldsymbol{x}$ by the known transforms [7, 25, 30]. Then we can put $\boldsymbol{\varphi}_{1}(\boldsymbol{y})=\tilde{\boldsymbol{x}}^{(1)}, \ldots, \boldsymbol{\varphi}_{p}(\boldsymbol{y})=\tilde{\boldsymbol{x}}^{(p)}$. In particular, one could choose $\boldsymbol{\varphi}_{1}(\boldsymbol{y})=\boldsymbol{y}$. In such a way, the vector $\boldsymbol{x}$ is pre-estimated from $\boldsymbol{y}$, and therefore, the overall $\boldsymbol{x}$ estimate by $\mathcal{T}_{p}$ will be improved. A new recursive method for finding $\tilde{\boldsymbol{x}}^{(1)}, \ldots, \tilde{\boldsymbol{x}}^{(p)}$ is given in Section 5.2.4 below.

Other particular cases of the proposed transform are considered in Sections 5.2.4 and 5.2.5.

Remark 1. The particular case of $\mathcal{T}_{p}$ considered in the item (iii) above can be interpreted as an operator form of the Fourier polynomial in Hilbert space [35]. The benefits associated with the Fourier polynomials are well known. In item (ii) of Section 5.2.5, this case is considered in more detail.

\section{Statement of the problem}

First, we define orthogonal and orthonormal operators as follows.

Definition 1. Let $\boldsymbol{u}_{k} \in L^{2}\left(\Omega, \mathbb{R}^{n}\right)$ and $\boldsymbol{v}_{k}=\mathcal{Q}_{k}\left(\boldsymbol{u}_{k}\right)$. The operators $\mathcal{Q}_{1}, \ldots, \mathcal{Q}_{p}$ are called pairwise orthonormal if $\mathbb{E}_{v_{i} v_{j}}=\left\{\begin{array}{ll}\mathbb{O}, & i \neq j, \\ I, & i=j\end{array}\right.$ for any $i, j=1, \ldots, p$. Here, $\mathbb{O}$ and $I$ are the zero matrix and identity matrix, respectively. If $\mathbb{E}_{v_{i} v_{j}}=\mathbb{O}$ for $i \neq j$ with $i, j=1, \ldots, p$, and if $\mathbb{E}_{v_{i} v_{j}}$ is not necessarily equal to $I$ for $i=j$ then $\mathcal{Q}_{1}, \ldots, \mathcal{Q}_{p}$ are called pairwise orthogonal.

Hereinafter, we suppose that $\mathcal{F}_{k}$ is linear for all $k=1, \ldots, p$ and that the Hilbert spaces are the finite dimensional Eucledian spaces, $H_{X}=\mathbb{R}^{m}$ and $H_{Y}=H_{k}=\tilde{H}_{k}=\mathbb{R}^{n}$. For any vector $\boldsymbol{g} \in L^{2}\left(\Omega, \mathbb{R}^{m}\right)$, we set

$$
E\left[\|\boldsymbol{g}\|^{2}\right]=\int_{\Omega}\|\boldsymbol{g}(\omega)\|^{2} d \mu(\omega)<\infty
$$

where $\|\boldsymbol{g}(\omega)\|$ is the Euclidean norm of $\boldsymbol{g}(\omega)$.

Let us denote

$$
J\left(f, \mathcal{F}_{1}, \ldots \mathcal{F}_{p}\right)=E\left[\left\|\boldsymbol{x}-\mathcal{T}_{p}(\boldsymbol{y})\right\|^{2}\right] .
$$

The problem is

(i) to find operators $\mathcal{Q}_{1}, \ldots, \mathcal{Q}_{p}$ satisfying Definition 1 , and

(ii) to determine the vector $f^{0}$ and operators $\mathcal{F}_{1}^{0}, \ldots, \mathcal{F}_{p}^{0}$ such that

$$
J\left(f^{0}, \mathcal{F}_{1}^{0}, \ldots \mathcal{F}_{p}^{0}\right)=\min _{f, \mathcal{F}_{1}, \ldots, \mathcal{F}_{p}} J\left(f, \mathcal{F}_{1}, \ldots, \mathcal{F}_{p}\right)
$$

subject to

$$
\operatorname{rank} \mathcal{F}_{1}=\eta_{1}, \quad \ldots, \quad \operatorname{rank} \mathcal{F}_{p}=\eta_{p}
$$

where $\eta_{1}+\cdots+\eta_{p}=\eta \leq \min \{m, n\}$. 
Here, for $k=1, \ldots, p$, (see, for example, [44])

$$
\operatorname{rank}\left(\mathcal{F}_{k}\right)=\operatorname{dim} \mathcal{F}_{k}\left(L^{2}\left(\Omega, \mathbb{R}^{n}\right)\right) .
$$

We write

$$
\mathcal{T}_{p}^{0}(\boldsymbol{y})=f^{0}+\sum_{k=1}^{p} \mathcal{F}_{k}^{0}\left(\boldsymbol{v}_{k}\right)
$$

with $\boldsymbol{v}_{k}$ defined by Definition 1 .

It is supposed that covariance matrices formed from vectors $\mathcal{Q}_{1} \boldsymbol{\varphi}_{1}(\boldsymbol{y}), \ldots, \mathcal{Q}_{p} \boldsymbol{\varphi}_{p}(\boldsymbol{y})$ in $(3)$ are known or can be estimated. Various estimation methods can be found in [36, 37, 38, 39, 40, 41]. We note that such an assumption is traditional $[1,2,3,4,5,6,7,8,9,10,11,12,13,14,15,16]$ in the study of optimal transforms. The effective estimate of covariance matrices represents a specific task [36, 37, 38, 39, 40,41] which is not considered in this paper.

Remark 2. Unlike known rank-constrained problems, we consider $p$ constraints (7). The number $p$ of the constraints and the ranks $\eta_{1}, \ldots, \eta_{p}$ form the degrees of freedom for $\mathcal{T}_{p}^{0}$. Variation of $p$ and $\eta_{1}, \ldots, \eta_{p}$ allows us to regulate accuracy associated with the transform $\mathcal{T}_{p}^{0}$ (see (21) in Section 5.2.1 and (46) in Section 5.2.2) and its compression ratio (see (67) in Section 5.2.4). It follows from (21) and (46) that the accuracy increases if $p$ and $\eta_{1}, \ldots, \eta_{p}$ increase. Conversely, by (67), the compression ratio is improved if $\eta_{1}, \ldots, \eta_{p}$ decrease.

\section{Solution of the problem}

The problem (6)-(7) generalises the known rank-constrained problems where only one constraint has been considered. Our plan for the solution is as follows. First, in Section 5.1, we will determine the operators $\mathcal{Q}_{1}, \ldots, \mathcal{Q}_{p}$. Then, in Section 5.2 , we will obtain $f^{0}$ and $\mathcal{F}_{1}^{0}, \ldots, \mathcal{F}_{p}^{0}$ satisfying (6) and (7).

\subsection{Determination of orthogonalizing operators $\mathcal{Q}_{1}, \ldots, \mathcal{Q}_{p}$}

If $M$ is a square matrix then we write $M^{1 / 2}$ for a matrix such that $M^{1 / 2} M^{1 / 2}=M$. We note that the matrix $M^{1 / 2}$ can be computed in various ways [42]. In this paper, $M^{1 / 2}$ is determined from the singular value decomposition (SVD) [43] of $M$.

For the case when matrix $\mathbb{E}_{v_{k} v_{k}}$ is invertible for any $k=1, \ldots, p$, the orthonormalization procedure is as follows. For $\boldsymbol{u}_{k} \in L^{2}\left(\Omega, \mathbb{R}^{n}\right)$, we write

$$
\left[\mathcal{Q}_{k}\left(\boldsymbol{u}_{k}\right)\right](\omega)=Q_{k} \boldsymbol{u}_{k}(\omega)
$$

where $Q_{k} \in \mathbb{R}^{n \times n}$. For $\boldsymbol{u}_{k}, \boldsymbol{v}_{j}, \boldsymbol{w}_{j} \in L^{2}\left(\Omega, \mathbb{R}^{n}\right)$, we also define operators $\mathcal{E}_{u_{k} v_{j}}, \mathcal{E}_{v_{j} v_{j}}^{-1}: L^{2}\left(\Omega, \mathbb{R}^{n}\right) \rightarrow$ $L^{2}\left(\Omega, \mathbb{R}^{n}\right)$ by the equations

$$
\left[\mathcal{E}_{u_{k} v_{j}}\left(\boldsymbol{w}_{j}\right)\right](\omega)=\mathbb{E}_{u_{k} v_{j}} \boldsymbol{w}_{j}(\omega) \quad \text { and } \quad\left[\mathcal{E}_{v_{j} v_{j}}^{-1}\left(\boldsymbol{w}_{j}\right)\right](\omega)=\mathbb{E}_{v_{j} v_{j}}^{-1} \boldsymbol{w}_{j}(\omega),
$$

respectively.

\section{Lemma 1. Let}

$$
\boldsymbol{w}_{1}=\boldsymbol{u}_{1} \quad \text { and } \quad \boldsymbol{w}_{i}=\boldsymbol{u}_{i}-\sum_{k=1}^{i-1} \mathcal{E}_{u_{i} w_{k}} \mathcal{E}_{w_{k} w_{k}}^{-1}\left(\boldsymbol{w}_{k}\right) \quad \text { for } \quad i=1, \ldots, p,
$$

where $\mathcal{E}_{w_{k} w_{k}}^{-1}$ exists. Then 
(i) the vectors $\boldsymbol{w}_{1}, \ldots, \boldsymbol{w}_{p}$ are pairwise orthogonal, and

(ii) the vectors $\boldsymbol{v}_{1}, \ldots, \boldsymbol{v}_{p}$, defined by

$$
\boldsymbol{v}_{i}=\mathcal{Q}_{i}\left(\boldsymbol{u}_{i}\right)
$$

with

$$
\mathcal{Q}_{i}\left(\boldsymbol{u}_{i}\right)=\left(\mathcal{E}_{w_{i} w_{i}}^{1 / 2}\right)^{-1}\left(\boldsymbol{w}_{i}\right)
$$

for $i=1, \ldots, p$, are pairwise orthonormal.

Proof. The proof is given in the Appendix.

For the case when matrix $\mathbb{E}_{v_{k} v_{k}}$ is singular for $k=1, \ldots, p$, the orthogonalizing operators $\mathcal{Q}_{1}, \ldots, \mathcal{Q}_{p}$ are determined by Lemma 3 below. Another difference from Lemma 1 is that the vectors $\boldsymbol{v}_{1}, \ldots, \boldsymbol{v}_{p}$ in Lemma 3 are pairwise orthogonal but not orthonormal. An intermediate result is given in Lemma 2.

The symbol $\dagger$ is used to denote the pseudo-inverse operator [45]. It is supposed that the pseudo-inverse $M^{\dagger}$ for matrix $M$ is determined from the SVD of $M$.

Lemma 2 ([26]). For any random vectors $\boldsymbol{g} \in L^{2}\left(\Omega, \mathbb{R}^{m}\right)$ and $\boldsymbol{h} \in L^{2}\left(\Omega, \mathbb{R}^{n}\right)$,

$$
\mathbb{E}_{g h} \mathbb{E}_{h h}^{\dagger} \mathbb{E}_{h h}=\mathbb{E}_{g h}
$$

Lemma 3. Let $\boldsymbol{v}_{i}=\mathcal{Q}_{i}\left(\boldsymbol{u}_{i}\right)$ for $i=1, \ldots, p$, where $\mathcal{Q}_{1}, \ldots, \mathcal{Q}_{p}$ are such that

$$
\mathcal{Q}_{1}\left(\boldsymbol{u}_{1}\right)=\boldsymbol{u}_{1} \quad \text { and } \quad \mathcal{Q}_{i}\left(\boldsymbol{u}_{i}\right)=\boldsymbol{u}_{i}-\sum_{k=1}^{i-1} \mathcal{Z}_{i k}\left(\boldsymbol{v}_{k}\right) \quad \text { for } \quad i=2, \ldots, p
$$

with $\mathcal{Z}_{i k}: L^{2}\left(\Omega, \mathbb{R}^{n}\right) \rightarrow L^{2}\left(\Omega, \mathbb{R}^{n}\right)$ defined by

$$
Z_{i k}=\mathbb{E}_{u_{i} v_{k}} \mathbb{E}_{v_{k} v_{k}}^{\dagger}+A_{i k}\left(I-\mathbb{E}_{v_{k} v_{k}} \mathbb{E}_{v_{k} v_{k}}^{\dagger}\right)
$$

with $A_{i k} \in \mathbb{R}^{n \times n}$ arbitrary. Then the vectors $\boldsymbol{v}_{1}, \ldots, \boldsymbol{v}_{p}$ are pairwise orthogonal.

Proof. The proof is given in the Appendix.

We note that Lemma 3 does not require invertibility of matrix $\mathbb{E}_{v_{k} v_{k}}$. At the same time, if $\mathbb{E}_{v_{k} v_{k}}^{-1}$ exists, then vectors $\boldsymbol{w}_{1}, \ldots, \boldsymbol{w}_{p}$ and $\boldsymbol{v}_{1}, \ldots, \boldsymbol{v}_{p}$ defined by (11) and Lemma 3 respectively, coincide.

Remark 3. Orthogonalization of random vectors is not, of course, a new idea. In particular, generalizations of the Gram-Schmidt orthogonalization procedure have been considered in [46, 47]. The proposed orthogonalization procedures in Lemmata 1 and 3 are different from those in $[46,47]$. In particular, Lemma 3 establishes the vector orthogonalization in terms of pseudoinverse operators. A particular case of the practical implementation of the random vector orthogonalization is considered in Section 6. 


\subsection{Determination of $f^{0}, \mathcal{F}_{1}^{0}, \ldots, \mathcal{F}_{p}^{0}$ satisfying $(6)-(7)$}

\subsubsection{The case when matrix $\mathbb{E}_{v_{i} v_{i}}$ is invertible for $i=1, \ldots, p$}

We consider the simpler case when $\mathbb{E}_{v_{i} v_{i}}$ is invertible for all $i=1, \ldots, p$. Then the vector $f^{0}$ and operators $\mathcal{F}_{1}^{0}, \ldots, \mathcal{F}_{p}^{0}$ satisfying (6)-(7) are defined from the following Theorem 1 . For each $i=1, \ldots, p$, let $U_{i} \Sigma_{i} V_{i}^{T}$ be the SVD of $\mathbb{E}_{x v_{i}}$,

$$
U_{i} \Sigma_{i} V_{i}^{T}=\mathbb{E}_{x v_{i}},
$$

where $U_{i} \in \mathbb{R}^{m \times n}, V_{i} \in \mathbb{R}^{n \times n}$ are orthogonal and $\Sigma_{i} \in \mathbb{R}^{n \times n}$ is diagonal,

$$
U_{i}=\left[s_{i 1}, \ldots, s_{i n}\right], \quad V_{i}=\left[d_{i 1}, \ldots, d_{i n}\right] \quad \text { and } \quad \Sigma_{i}=\operatorname{diag}\left(\alpha_{i 1}, \ldots, \alpha_{i n}\right)
$$

with $\alpha_{i 1} \geq \cdots \geq \alpha_{i r}>0, \alpha_{i, r+1}=\cdots=\alpha_{i n}=0$ and $r=1, \ldots, n$ where $r=r(i)$. We set

$$
U_{i \eta_{i}}=\left[s_{i 1}, \ldots, s_{i \eta_{i}}\right], \quad V_{i \eta_{i}}=\left[d_{i 1}, \ldots, d_{i \eta_{i}}\right] \quad \text { and } \quad \Sigma_{i \eta_{i}}=\operatorname{diag}\left(\alpha_{i 1}, \ldots, \alpha_{i \eta_{i}}\right) \text {, }
$$

where $U_{i \eta_{i}} \in \mathbb{R}^{m \times \eta_{i}}, V_{i \eta_{i}} \in \mathbb{R}^{n \times \eta_{i}}$ and $\Sigma_{i \eta_{i}} \in \mathbb{R}^{\eta_{i} \times \eta_{i}}$. Now we define $K_{i \eta_{i}} \in \mathbb{R}^{m \times n}$ and $\mathcal{K}_{i \eta_{i}}$ : $L^{2}\left(\Omega, \mathbb{R}^{n}\right) \rightarrow L^{2}\left(\Omega, \mathbb{R}^{m}\right)$ by

$$
K_{i \eta_{i}}=U_{i \eta_{i}} \Sigma_{i \eta_{i}} V_{i \eta_{i}}^{T} \quad \text { and } \quad\left[\mathcal{K}_{i \eta_{i}}\left(\boldsymbol{w}_{i}\right)\right](\omega)=K_{i \eta_{i}}\left[\boldsymbol{w}_{i}(\omega)\right],
$$

respectively, for any $\boldsymbol{w}_{i} \in L^{2}\left(\Omega, \mathbb{R}^{n}\right)$.

Theorem 1. Let $\boldsymbol{v}_{1}, \ldots, \boldsymbol{v}_{p}$ be determined by Lemma 1. Then the vector $f^{0}$ and operators $\mathcal{F}_{1}^{0}, \ldots, \mathcal{F}_{p}^{0}$, satisfying $(6)-(7)$, are determined by

$$
f^{0}=E[\boldsymbol{x}]-\sum_{k=1}^{p} F_{k}^{0} E\left[\boldsymbol{v}_{k}\right] \quad \text { and } \quad \mathcal{F}_{1}^{0}=\mathcal{K}_{1 \eta_{1}}, \quad \ldots, \quad \mathcal{F}_{p}^{0}=\mathcal{K}_{p \eta_{p}} .
$$

The accuracy associated with transform $\mathcal{T}_{p}^{0}$, determined by (8) and (20), is given by

$$
E\left[\left\|\boldsymbol{x}-\mathcal{T}_{p}^{0}(\boldsymbol{y})\right\|^{2}\right]=\left\|\mathbb{E}_{x x}^{1 / 2}\right\|^{2}-\sum_{k=1}^{p} \sum_{j=1}^{\eta_{k}} \alpha_{k j}^{2} .
$$

Proof. The functional $J\left(f, \mathcal{F}_{1}, \ldots, \mathcal{F}_{p}\right)$ is written as

$$
\begin{aligned}
J\left(f, \mathcal{F}_{1}, \ldots, \mathcal{F}_{p}\right)= & \operatorname{tr}\left[E_{x x}-E[\boldsymbol{x}] f^{T}-\sum_{i=1}^{p} E_{x v_{i}} F_{i}^{T}-f E\left[\boldsymbol{x}^{T}\right]+f f^{T}+f \sum_{i=1}^{p} E\left[\boldsymbol{v}_{i}^{T}\right] F_{i}^{T}\right. \\
& \left.-\sum_{i=1}^{p} F_{i} E_{v_{i} x}+\sum_{i=1}^{p} F_{i} E\left[\boldsymbol{v}_{i}\right] f^{T}+E\left(\sum_{i=1}^{p} \mathcal{F}_{i}\left(\boldsymbol{v}_{i}\right)\left[\sum_{k=1}^{p} \mathcal{F}_{i}\left(\boldsymbol{v}_{i}\right)\right]^{T}\right)\right] .
\end{aligned}
$$

We remind (see Section 2) that here and below, $F_{i}$ is defined by $\left[\mathcal{F}_{i}\left(\boldsymbol{v}_{i}\right)\right](\omega)=F_{i}\left[\boldsymbol{v}_{i}(\omega)\right]$ so that, for example, $E\left[\mathcal{F}_{k}\left(\boldsymbol{v}_{k}\right) \boldsymbol{x}_{k}^{T}\right]=F_{k} E_{v_{k} x_{k}}$. In other words, the right hand side in (22) is a function of $f, \mathcal{F}_{1}, \ldots, \mathcal{F}_{p}$.

Let us show that $J\left(f, \mathcal{F}_{1}, \ldots, \mathcal{F}_{p}\right)$ can be represented as

$$
J\left(f, \mathcal{F}_{1}, \ldots, \mathcal{F}_{p}\right)=J_{0}+J_{1}+J_{2},
$$

where

$$
J_{0}=\left\|\mathbb{E}_{x x}^{1 / 2}\right\|^{2}-\sum_{i=1}^{p}\left\|\mathbb{E}_{x v_{i}}\right\|^{2},
$$




$$
J_{1}=\left\|f-E[\boldsymbol{x}]+\sum_{i=1}^{p} F_{i} E\left[\boldsymbol{v}_{i}\right]\right\|^{2} \quad \text { and } \quad J_{2}=\sum_{i=1}^{p}\left\|F_{i}-\mathbb{E}_{x v_{i}}\right\|^{2} .
$$

Indeed, $J_{1}$ and $J_{2}$ are rewritten as follows

$$
\begin{aligned}
J_{1}= & \operatorname{tr}\left(f f^{T}-f E\left[\boldsymbol{x}^{T}\right]+\sum_{i=1}^{p} f E\left[\boldsymbol{v}_{i}^{T}\right] F_{i}+E[\boldsymbol{x}] E\left[\boldsymbol{x}^{T}\right]-E[\boldsymbol{x}] f^{T}-\sum_{i=1}^{p} E[\boldsymbol{x}] E\left[\boldsymbol{v}_{i}^{T}\right] F_{i}^{T}\right. \\
& \left.+\sum_{i=1}^{p} F_{i} E\left[\boldsymbol{v}_{i}\right] f^{T}-\sum_{i=1}^{p} F_{i} E\left[\boldsymbol{v}_{i}\right] E\left[\boldsymbol{x}^{T}\right]+\sum_{i=1}^{p} F_{i} E\left[\boldsymbol{v}_{i}\right] \sum_{k=1}^{p} E\left[\boldsymbol{v}_{k}^{T}\right] F_{k}^{T}\right)
\end{aligned}
$$

and

$$
J_{2}=\sum_{i=1}^{p} \operatorname{tr}\left(F_{i}-\mathbb{E}_{x v_{i}}\right)\left(F_{i}^{T}-\mathbb{E}_{v_{i} x}\right)=\sum_{i=1}^{p} \operatorname{tr}\left(F_{i} F_{i}^{T}-F_{i} \mathbb{E}_{v_{i} x}-\mathbb{E}_{x v_{i}} F_{i}^{T}+\mathbb{E}_{x v_{i}} \mathbb{E}_{v_{i} x}\right) .
$$

In (27), $\sum_{i=1}^{p} \operatorname{tr}\left(F_{i} F_{i}^{T}\right)$ can be represented in the form

$$
\sum_{i=1}^{p} \operatorname{tr}\left(F_{i} F_{i}^{T}\right)=\operatorname{tr}\left[E\left(\sum_{i=1}^{p} F_{i} \boldsymbol{v}_{i} \sum_{k=1}^{p} \boldsymbol{v}_{k}^{T} F_{k}^{T}\right)\right]-\operatorname{tr}\left(\sum_{i=1}^{p} F_{i} E\left[\boldsymbol{v}_{i}\right] \sum_{k=1}^{p} E\left[\boldsymbol{v}_{k}^{T}\right] F_{k}^{T}\right)
$$

because

$$
E\left[\boldsymbol{v}_{i} \boldsymbol{v}_{k}^{T}\right]-E\left[\boldsymbol{v}_{i}\right] E\left[\boldsymbol{v}_{k}^{T}\right]= \begin{cases}\mathbb{O}, & i \neq k \\ I, & i=k\end{cases}
$$

due to the orthonormality of vectors $\boldsymbol{v}_{1}, \ldots, \boldsymbol{v}_{p}$.

Then

$$
\begin{aligned}
J_{0}+J_{1}+J_{2}= & \operatorname{tr}\left(E_{x x}-E[\boldsymbol{x}] E\left[\boldsymbol{x}^{T}\right]\right)-\sum_{i=1}^{p} \operatorname{tr}\left[\mathbb{E}_{x v_{i}} \mathbb{E}_{v_{i} x}\right] \\
& +\operatorname{tr}\left(f f^{T}-f E\left[\boldsymbol{x}^{T}\right]+\sum_{i=1}^{p} f E\left[\boldsymbol{v}_{i}^{T}\right] F_{i}+E[\boldsymbol{x}] E\left[\boldsymbol{x}^{T}\right]-E[\boldsymbol{x}] f^{T}\right. \\
& -\sum_{i=1}^{p} E[\boldsymbol{x}] E\left[\boldsymbol{v}_{i}^{T}\right] F_{i}^{T}+\sum_{i=1}^{p} F_{i} E\left[\boldsymbol{v}_{i}\right] f^{T}-\sum_{i=1}^{p} F_{i} E\left[\boldsymbol{v}_{i}\right] E\left[\boldsymbol{x}^{T}\right] \\
& \left.+\sum_{i=1}^{p} F_{i} E\left[\boldsymbol{v}_{i}\right] \sum_{k=1}^{p} E\left[\boldsymbol{v}_{k}^{T}\right] F_{k}^{T}\right)+\operatorname{tr}\left[E\left(\sum_{i=1}^{p} F_{i} \boldsymbol{v}_{i} \sum_{k=1}^{p} \boldsymbol{v}_{k}^{T} F_{k}^{T}\right)\right] \\
& -\operatorname{tr}\left(\sum_{i=1}^{p} F_{i} E\left[\boldsymbol{v}_{i}\right] \sum_{k=1}^{p} E\left[\boldsymbol{v}_{k}^{T}\right] F_{k}^{T}\right) \\
& -\sum_{i=1}^{p} \operatorname{tr}\left(F_{i} E_{v_{i} x}-F_{i} E\left[\boldsymbol{v}_{i}\right] E\left[\boldsymbol{x}^{T}\right]+E_{x v_{i}} F_{i}^{T}-E[\boldsymbol{x}] E\left[\boldsymbol{v}_{i}^{T}\right] F_{i}^{T}-\mathbb{E}_{x v_{i}} \mathbb{E}_{v_{i} x}\right) \\
= & J\left(f, \mathcal{F}_{1}, \ldots, \mathcal{F}_{p}\right) .
\end{aligned}
$$

Hence, (23) is true. Therefore,

$$
J\left(f, \mathcal{F}_{1}, \ldots, \mathcal{F}_{p}\right)=\left\|\mathbb{E}_{x x}^{1 / 2}\right\|^{2}-\sum_{k=1}^{p}\left\|\mathbb{E}_{x v_{k}}\right\|^{2}+\left\|f-E[\boldsymbol{x}]+\sum_{k=1}^{p} F_{k} E\left[\boldsymbol{v}_{k}\right]\right\|^{2}
$$




$$
+\sum_{k=1}^{p}\left\|F_{k}-\mathbb{E}_{x v_{k}}\right\|^{2}
$$

It follows from (32) that the constrained minimum (6)-(7) is achieved if $f=f^{0}$ with $f^{0}$ given by (20), and if $F_{k}^{0}$ is such that

$$
J_{k}\left(F_{k}^{0}\right)=\min _{F_{k}} J_{k}\left(F_{k}\right) \quad \text { subject to } \operatorname{rank}\left(F_{k}\right)=\eta_{k},
$$

where $J_{k}\left(F_{k}\right)=\left\|F_{k}-\mathbb{E}_{x v_{k}}\right\|^{2}$. The solution to (33) is given [43] by

$$
F_{k}^{0}=K_{k \eta_{k}}
$$

Then

$$
E\left[\left\|\boldsymbol{x}-\mathcal{T}_{p}^{0}(\boldsymbol{y})\right\|^{2}\right]=\left\|\mathbb{E}_{x x}^{1 / 2}\right\|^{2}-\sum_{k=1}^{p}\left(\left\|\mathbb{E}_{x v_{k}}\right\|^{2}-\left\|K_{k \eta_{k}}-\mathbb{E}_{x v_{k}}\right\|^{2}\right) .
$$

Here [43],

$$
\left\|\mathbb{E}_{x v_{k}}\right\|^{2}=\sum_{j=1}^{r} \alpha_{k j}^{2} \quad \text { and } \quad\left\|K_{k \eta_{k}}-\mathbb{E}_{x v_{k}}\right\|^{2}=\sum_{j=\eta_{k}+1}^{r} \alpha_{k j}^{2}
$$

with $r=r(k)$. Thus, $(21)$ is true. The theorem is proved.

Corollary 1. Let $\boldsymbol{v}_{1}, \ldots, \boldsymbol{v}_{p}$ be determined by Lemma 1 . Then the vector $\hat{f}$ and operators $\hat{\mathcal{F}}_{1}, \ldots, \hat{\mathcal{F}}_{p}$ satisfying the unconstrained problem (6), are determined by

$$
\hat{f}=E[\boldsymbol{x}]-\sum_{k=1}^{p} \hat{F}_{k} E\left[\boldsymbol{v}_{k}\right] \quad \text { and } \quad \hat{\mathcal{F}}_{1}=\mathcal{E}_{x v_{1}}, \quad \ldots, \quad \hat{\mathcal{F}}_{p}=\mathcal{E}_{x v_{p}}
$$

with $\hat{\mathcal{F}}_{k}$ such that $\left[\hat{\mathcal{F}}_{k}\left(\boldsymbol{v}_{k}\right)\right](\omega)=\hat{F}_{k} \boldsymbol{v}_{k}(\omega)$ where $\hat{F}_{k} \in \mathbb{R}^{n \times m}$ and $k=1, \ldots, p$.

The accuracy associated with transform $\hat{\mathcal{T}}_{p}$ given by

$$
\hat{\mathcal{T}}_{p}(\boldsymbol{y})=\hat{f}+\sum_{k=1}^{p} \hat{\mathcal{F}}_{k}\left(\boldsymbol{v}_{k}\right)
$$

is such that

$$
E\left[\left\|\boldsymbol{x}-\hat{\mathcal{T}}_{p}(\boldsymbol{y})\right\|^{2}\right]=\left\|\mathbb{E}_{x x}^{1 / 2}\right\|^{2}-\sum_{k=1}^{p}\left\|\mathbb{E}_{x v_{k}}\right\|^{2} .
$$

Proof. The proof follows directly from (32).

\subsubsection{The case when matrix $\mathbb{E}_{v_{k} v_{k}}$ is not invertible for $k=1, \ldots, p$}

We write $A_{k} \in \mathbb{R}^{m \times n}$ for an arbitrary matrix, and define operators $\mathcal{A}_{k}: L^{2}\left(\Omega, \mathbb{R}^{n}\right) \rightarrow L^{2}\left(\Omega, \mathbb{R}^{m}\right)$ and $\mathcal{E}_{v_{k} v_{k}}, \mathcal{E}_{v_{k} v_{k}}^{\dagger},\left(\mathcal{E}_{v_{k} v_{k}}^{1 / 2}\right)^{\dagger}: L^{2}\left(\Omega, \mathbb{R}^{n}\right) \rightarrow L^{2}\left(\Omega, \mathbb{R}^{n}\right)$ similarly to those in (9) and (10).

For the case under consideration (matrix $\mathbb{E}_{v_{k} v_{k}}$ is not invertible), we introduce the SVD of $\mathbb{E}_{x v_{k}}\left(\mathbb{E}_{v_{k} v_{k}}^{1 / 2}\right)^{\dagger}$,

$$
U_{k} \Sigma_{k} V_{k}^{T}=\mathbb{E}_{x v_{k}}\left(\mathbb{E}_{v_{k} v_{k}}^{1 / 2}\right)^{\dagger},
$$


where, as above, $U_{k} \in \mathbb{R}^{m \times n}, V_{k} \in \mathbb{R}^{n \times n}$ are orthogonal and $\Sigma_{k} \in \mathbb{R}^{n \times n}$ is diagonal,

$$
U_{k}=\left[s_{k 1}, \ldots, s_{k n}\right], \quad V_{k}=\left[d_{k 1}, \ldots, d_{k n}\right] \quad \text { and } \quad \Sigma_{k}=\operatorname{diag}\left(\beta_{k 1}, \ldots, \beta_{k n}\right)
$$

with $\beta_{k 1} \geq \cdots \geq \beta_{k r}>0, \beta_{k, r+1}=\cdots=\beta_{k n}=0, r=1, \ldots, n$ and $r=r(k)$.

Let us set

$$
\begin{aligned}
U_{k \eta_{k}} & =\left[s_{k 1}, \ldots, s_{k \eta_{k}}\right], \quad V_{k \eta_{k}}=\left[d_{k 1}, \ldots, d_{k \eta_{k}}\right] \quad \text { and } \\
\Sigma_{k \eta_{k}} & =\operatorname{diag}\left(\beta_{k 1}, \ldots, \beta_{k \eta_{k}}\right),
\end{aligned}
$$

where $U_{k \eta_{k}} \in \mathbb{R}^{m \times \eta_{k}}, V_{k \eta_{k}} \in \mathbb{R}^{n \times \eta_{k}}$ and $\Sigma_{k \eta_{k}} \in \mathbb{R}^{\eta_{k} \times \eta_{k}}$. Now we define $G_{k \eta_{k}} \in \mathbb{R}^{m \times n}$ and $\mathcal{G}_{k \eta_{k}}: L^{2}\left(\Omega, \mathbb{R}^{n}\right) \rightarrow L^{2}\left(\Omega, \mathbb{R}^{m}\right)$ by

$$
G_{k \eta_{k}}=U_{k \eta_{k}} \Sigma_{k \eta_{k}} V_{k \eta_{k}}^{T} \quad \text { and } \quad\left[\mathcal{G}_{k \eta_{k}}\left(\boldsymbol{w}_{k}\right)\right](\omega)=G_{k \eta_{k}}\left[\boldsymbol{w}_{k}(\omega)\right],
$$

respectively, for any $\boldsymbol{w}_{k} \in L^{2}\left(\Omega, \mathbb{R}^{n}\right)$.

As noted before, we write $\mathcal{I}$ for the identity operator.

Theorem 2. Let $\boldsymbol{v}_{1}, \ldots, \boldsymbol{v}_{p}$ be determined by Lemma 3. Then $f^{0}$ and $\mathcal{F}_{1}^{0}, \ldots, \mathcal{F}_{p}^{0}$, satisfying (6)-(7), are determined by

$$
f^{0}=E[\boldsymbol{x}]-\sum_{k=1}^{p} F_{k}^{0} E\left[\boldsymbol{v}_{k}\right]
$$

and

$$
\begin{aligned}
& \mathcal{F}_{1}^{0}=\mathcal{G}_{1 \eta_{1}}\left(\mathcal{E}_{v_{1} v_{1}}^{1 / 2}\right)^{\dagger}+\mathcal{A}_{1}\left[\mathcal{I}-\mathcal{E}_{v_{1} v_{1}}^{1 / 2}\left(\mathcal{E}_{v_{1} v_{1}}^{1 / 2}\right)^{\dagger}\right], \\
& \ldots \ldots \ldots \ldots \ldots \ldots \ldots \ldots \ldots \ldots \ldots \ldots \ldots \\
& \mathcal{F}_{p}^{0}=\mathcal{G}_{p \eta_{p}}\left(\mathcal{E}_{v_{p} v_{p}}^{1 / 2}\right)^{\dagger}+\mathcal{A}_{p}\left[\mathcal{I}-\mathcal{E}_{v_{p} v_{p}}^{1 / 2}\left(\mathcal{E}_{v_{p} v_{p}}^{1 / 2}\right)^{\dagger}\right],
\end{aligned}
$$

where for $k=1, \ldots, p, \mathcal{A}_{k}$ is any linear operator such that $\operatorname{rank} \mathcal{F}_{k}^{0} \leq \eta_{k}{ }^{1}$.

The accuracy associated with transform $\mathcal{T}_{p}^{0}$ given by (8) and $(43)-(45)$ is such that

$$
E\left[\left\|\boldsymbol{x}-\mathcal{T}_{p}^{0}(\boldsymbol{y})\right\|^{2}\right]=\left\|\mathbb{E}_{x x}^{1 / 2}\right\|^{2}-\sum_{k=1}^{p} \sum_{j=1}^{\eta_{k}} \beta_{k j}^{2} .
$$

Proof. For $\boldsymbol{v}_{1}, \ldots, \boldsymbol{v}_{p}$ determined by Lemma $3, J\left(f, \mathcal{F}_{1}, \ldots, \mathcal{F}_{p}\right)$ is represented by (22) as well. Let us consider $J_{0}, J_{1}$ and $J_{2}$ given by

$$
\begin{aligned}
& J_{0}=\left\|\mathbb{E}_{x x}^{1 / 2}\right\|^{2}-\sum_{k=1}^{p}\left\|\mathbb{E}_{x v_{k}}\left(\mathbb{E}_{v_{k} v_{k}}^{1 / 2}\right)^{\dagger}\right\|^{2}, \\
& J_{1}=\left\|f-E[\boldsymbol{x}]+\sum_{k=1}^{p} F_{k} E\left[\boldsymbol{v}_{k}\right]\right\|^{2} \quad \text { and } \quad J_{2}=\sum_{k=1}^{p}\left\|F_{k} \mathbb{E}_{v_{k} v_{k}}^{1 / 2}-\mathbb{E}_{x v_{k}}\left(\mathbb{E}_{v_{k} v_{k}}^{1 / 2}\right)^{\dagger}\right\|^{2} .
\end{aligned}
$$

To show that

$$
J\left(f, \mathcal{F}_{1}, \ldots, \mathcal{F}_{p}\right)=J_{0}+J_{1}+J_{2}
$$

with $J\left(f, \mathcal{F}_{1}, \ldots, \mathcal{F}_{p}\right)$ defined by $(22)$, we use the relationships (see [26])

$$
\mathbb{E}_{x v_{k}} \mathbb{E}_{v_{k} v_{k}}^{\dagger} \mathbb{E}_{v_{k} v_{k}}=\mathbb{E}_{x v_{k}} \quad \text { and } \quad \mathbb{E}_{v_{k} v_{k}}^{\dagger} \mathbb{E}_{v_{k} v_{k}}^{1 / 2}=\left(\mathbb{E}_{v_{k} v_{k}}^{1 / 2}\right)^{\dagger}
$$

\footnotetext{
${ }^{1}$ In particular, $\mathcal{A}_{k}$ can be chosen as the zero operator.
} 
Then

$$
\begin{aligned}
J_{1}= & \operatorname{tr}\left(f f^{T}-f E\left[\boldsymbol{x}^{T}\right]+\sum_{k=1}^{p} f E\left[\boldsymbol{v}_{k}^{T}\right] F_{k}+E[\boldsymbol{x}] E\left[\boldsymbol{x}^{T}\right]-E[\boldsymbol{x}] f^{T}-\sum_{k=1}^{p} E[\boldsymbol{x}] E\left[\boldsymbol{v}_{k}^{T}\right] F_{k}^{T}\right. \\
& \left.+\sum_{k=1}^{p} F_{k} E\left[\boldsymbol{v}_{k}\right] f^{T}-\sum_{k=1}^{p} F_{k} E\left[\boldsymbol{v}_{k}\right] E\left[\boldsymbol{x}^{T}\right]+\sum_{k=1}^{p} F_{k} E\left[\boldsymbol{v}_{k}\right] \sum_{i=1}^{p} E\left[\boldsymbol{v}_{i}^{T}\right] F_{i}^{T}\right)
\end{aligned}
$$

and

$$
\begin{aligned}
J_{2} & =\sum_{k=1}^{p} \operatorname{tr}\left(F_{k}-\mathbb{E}_{x v_{k}} \mathbb{E}_{v_{k} v_{k}}^{\dagger}\right) \mathbb{E}_{v_{k} v_{k}}\left(F_{k}^{T}-\mathbb{E}_{v_{k} v_{k}}^{\dagger} \mathbb{E}_{v_{k} x}\right) \\
& =\sum_{k=1}^{p} \operatorname{tr}\left(F_{k} \mathbb{E}_{v_{k} v_{k}} F_{k}^{T}-F_{k} \mathbb{E}_{v_{k} x}-\mathbb{E}_{x v_{k}} F_{k}^{T}+\mathbb{E}_{x v_{k}} \mathbb{E}_{v_{k} v_{k}}^{\dagger} \mathbb{E}_{v_{k} x}\right),
\end{aligned}
$$

where

$$
\sum_{k=1}^{p} \operatorname{tr}\left(F_{k} \mathbb{E}_{v_{k} v_{k}} F_{k}^{T}\right)=\operatorname{tr}\left[E\left(\sum_{k=1}^{p} F_{k} \boldsymbol{v}_{k} \sum_{i=1}^{p} \boldsymbol{v}_{i}^{T} F_{i}^{T}\right)\right]-\operatorname{tr}\left(\sum_{k=1}^{p} F_{k} E\left[\boldsymbol{v}_{k}\right] \sum_{i=1}^{p} E\left[\boldsymbol{v}_{i}^{T}\right] F_{i}^{T}\right)
$$

because

$$
E\left[\boldsymbol{v}_{i} \boldsymbol{v}_{k}^{T}\right]-E\left[\boldsymbol{v}_{i}\right] E\left[\boldsymbol{v}_{k}^{T}\right]=\mathbb{O} \quad \text { for } \quad i \neq k
$$

due to orthogonality of the vectors $\boldsymbol{v}_{1}, \ldots, \boldsymbol{v}_{s}$. On the basis of (50)-(53) and similarly to (30)(31), we establish that (49) is true. Hence,

$$
\begin{aligned}
J\left(f, \mathcal{F}_{1}, \ldots, \mathcal{F}_{p}\right)= & \left\|\mathbb{E}_{x x}^{1 / 2}\right\|^{2}-\sum_{k=1}^{p}\left\|\mathbb{E}_{x v_{k}}\left(\mathbb{E}_{v_{k} v_{k}}^{1 / 2}\right)^{\dagger}\right\|^{2}+\| f-E[\boldsymbol{x}] \\
& +\sum_{k=1}^{p} F_{k} E\left[\boldsymbol{v}_{k}\right]\left\|^{2}+\sum_{k=1}^{p}\right\| F_{k} \mathbb{E}_{v_{k} v_{k}}^{1 / 2}-\mathbb{E}_{x v_{k}}\left(\mathbb{E}_{v_{k} v_{k}}^{1 / 2}\right)^{\dagger} \|^{2} .
\end{aligned}
$$

It follows from the last two terms in (55) that the constrained minimum (6)-(7) is achieved if $f=f^{0}$ with $f^{0}$ given by (43), and $F_{k}^{0}$ is such that

$$
J_{k}\left(F_{k}^{0}\right)=\min _{F_{k}} J_{k}\left(F_{k}\right) \quad \text { subject to } \operatorname{rank}\left(F_{k}\right)=\eta_{k}
$$

where $J_{k}\left(F_{k}\right)=\left\|F_{k} \mathbb{E}_{v_{k} v_{k}}^{1 / 2}-\mathbb{E}_{x v_{k}}\left(\mathbb{E}_{v_{k} v_{k}}^{1 / 2}\right)^{\dagger}\right\|^{2}$. The constrained minimum (6)-(7) is achieved if $f=f^{0}$ is defined by (43), and if [43]

$$
F_{k} \mathbb{E}_{v_{k} v_{k}}^{1 / 2}=G_{\eta_{k}} .
$$

The matrix equation (57) has the general solution [45]

$$
F_{k}=F_{k}^{0}=G_{\eta_{k}}\left(\mathbb{E}_{v_{k} v_{k}}^{1 / 2}\right)^{\dagger}+A_{k}\left[I-\mathbb{E}_{v_{k} v_{k}}^{1 / 2}\left(\mathbb{E}_{v_{k} v_{k}}^{1 / 2}\right)^{\dagger}\right]
$$

if and only if

$$
G_{\eta_{k}}\left(\mathbb{E}_{v_{k} v_{k}}^{1 / 2}\right)^{\dagger} \mathbb{E}_{v_{k} v_{k}}^{1 / 2}=G_{\eta_{k}}
$$

The latter is satisfied on the basis of the following derivation ${ }^{2}$.

\footnotetext{
${ }^{2}$ Note that the matrix $I-\mathbb{E}_{v_{k} v_{k}}^{1 / 2}\left(\mathbb{E}_{v_{k} v_{k}}^{1 / 2}\right)^{\dagger}$ is simply a projection onto the null space of $\mathbb{E}_{v_{k} v_{k}}$ and can be replaced by $I-\mathbb{E}_{v_{k} v_{k}}\left(\mathbb{E}_{v_{k} v_{k}}\right)^{\dagger}$.
} 
As an extension of the technique presented in the proving Lemmata 1 and 2 in [26], it can be shown that for any matrices $Q_{1}, Q_{2} \in \mathbb{R}^{m \times n}$,

$$
\mathcal{N}\left(Q_{1}\right) \subseteq \mathcal{N}\left(Q_{2}\right) \quad \Rightarrow \quad Q_{2}\left(I-Q_{1}^{\dagger} Q_{1}\right)=\mathbb{O},
$$

where $\mathcal{N}\left(Q_{i}\right)$ is the null space of $Q_{i}$ for $i=1,2$. In regard of the equation under consideration,

$$
\mathcal{N}\left(\left[\mathbb{E}_{v_{k} v_{k}}^{1 / 2}\right]^{\dagger}\right) \subseteq \mathcal{N}\left(\mathbb{E}_{x v_{k}}\left[\mathbb{E}_{v_{k} v_{k}}^{1 / 2}\right]^{\dagger}\right)
$$

The definition of $G_{\eta_{k}}$ implies that

$$
\mathcal{N}\left(\mathbb{E}_{x v_{k}}\left[\mathbb{E}_{v_{k} v_{k}}^{1 / 2}\right]^{\dagger}\right) \subseteq \mathcal{N}\left(G_{\eta_{k}}\right) \quad \text { and then } \quad \mathcal{N}\left(\left[\mathbb{E}_{v_{k} v_{k}}^{1 / 2}\right]^{\dagger}\right) \subseteq \mathcal{N}\left(G_{\eta_{k}}\right)
$$

On the basis of (60), the latter implies $G_{\eta_{k}}\left[I-\left(\mathbb{E}_{v_{k} v_{k}}^{1 / 2}\right)^{\dagger} \mathbb{E}_{v_{k} v_{k}}^{1 / 2}\right]=\mathbb{O}$, i.e. (59) is true. Hence, (58) and (44)-(45) are true as well.

Next, similar to (35),

$$
\left\|\mathbb{E}_{x v_{k}}\left(\mathbb{E}_{v_{k} v_{k}}^{1 / 2}\right)^{\dagger}\right\|^{2}-\left\|G_{\eta_{k}}-\mathbb{E}_{x v_{k}}\left(\mathbb{E}_{v_{k} v_{k}}^{1 / 2}\right)^{\dagger}\right\|^{2}=\sum_{j=1}^{\eta_{k}} \beta_{k j}^{2}
$$

Then (46) follows from (55), (58), (43) and (62).

Remark 4. The known reduced-rank transforms based on the Volterra polynomial structure [16, $27,29]$ require the computation of a covariance matrix similar to $\mathbb{E}_{v v}$, where $\boldsymbol{v}=\left[\boldsymbol{v}_{1}, \ldots, \boldsymbol{v}_{p}\right]^{T}$, but for $p=N$ where $N$ is large (see Sections 1 and 2). The relationships (30)-(33) and (51)-(56) illustrate the nature of the proposed method and its difference from the techniques in $[16,27,29]$ : due to the structure $(3)$ of the transform $\mathcal{T}_{p}$, the procedure for finding $f^{0}, \mathcal{F}_{1}^{0}, \ldots, \mathcal{F}_{p}^{0}$ avoids direct computation of $\mathbb{E}_{v v}$ which could be troublesome due to large $N$. If operators $\mathcal{Q}_{1}, \ldots, \mathcal{Q}_{p}$ are orthonormal, as in Theorem 1 , then $(29)$ is true and the covariance matrix $\mathbb{E}_{v v}$ is reduced to the identity. If operators $\mathcal{Q}_{1}, \ldots, \mathcal{Q}_{p}$ are orthogonal, as in Theorem 2, then (54) holds and the covariance matrix $\mathbb{E}_{v v}$ is reduced to a block-diagonal form with non-zero blocks $\mathbb{E}_{v_{1} v_{1}}, \ldots, \mathbb{E}_{v_{p} v_{p}}$ so that

$$
\mathbb{E}_{v v}=\left[\begin{array}{cccc}
\mathbb{E}_{v_{1} v_{1}} & \mathbb{O} & \ldots & \mathbb{O} \\
\mathbb{O} & \mathbb{E}_{v_{2} v_{2}} & \ldots & \mathbb{O} \\
\ldots & \ldots & \ldots & \ldots \\
\mathbb{O} & \mathbb{O} & \ldots & \mathbb{E}_{v_{p} v_{p}}
\end{array}\right]
$$

with $\mathbb{O}$ denoting the zero block. As a result, the procedure for finding $f^{0}, \mathcal{F}_{1}^{0}, \ldots, \mathcal{F}_{p}^{0}$ is reduced to $p$ separate rank-constrained problems (33) or (56). Unlike the methods in [16, 27, 29], the operators $\mathcal{F}_{1}^{0}, \ldots, \mathcal{F}_{0}^{p}$ are determined with much smaller $m \times n$ and $n \times n$ matrices given by the simple formulae (20) and (43)-(45). This implies a reduction in computational work compared with that required by the approach in $[27,29,34]$.

Corollary 2. Let $\boldsymbol{v}_{1}, \ldots, \boldsymbol{v}_{p}$ be determined by Lemma 3. Then the vector $\bar{f}$ and operators $\overline{\mathcal{F}}_{1}, \ldots, \overline{\mathcal{F}}_{p}$, satisfying the unconstrained minimum (6), are determined by

$$
\bar{f}=E[\boldsymbol{x}]-\sum_{k=1}^{p} \bar{F}_{k} E\left[\boldsymbol{v}_{k}\right]
$$

and

$$
\overline{\mathcal{F}}_{1}=\mathcal{E}_{x v_{1}} \mathcal{E}_{v_{1} v_{1}}^{\dagger}+\mathcal{A}_{1}\left[\mathcal{I}-\mathcal{E}_{v_{1} v_{1}} \mathcal{E}_{v_{1} v_{1}}^{\dagger}\right]
$$




$$
\overline{\mathcal{F}}_{p}=\mathcal{E}_{x v_{p}} \mathcal{E}_{v_{p} v_{p}}^{\dagger}+\mathcal{A}_{p}\left[\mathcal{I}-\mathcal{E}_{v_{p} v_{p}} \mathcal{E}_{v_{p} v_{p}}^{\dagger}\right]
$$

The associated accuracy for transform $\overline{\mathcal{T}}_{p}$, defined by

$$
\overline{\mathcal{T}}_{p}(\boldsymbol{y})=\bar{f}+\sum_{k=1}^{p} \overline{\mathcal{F}}_{k}\left(\boldsymbol{v}_{k}\right)
$$

is given by

$$
E\left[\left\|\boldsymbol{x}-\overline{\mathcal{T}}_{p}(\boldsymbol{y})\right\|^{2}\right]=\left\|\mathbb{E}_{x x}^{1 / 2}\right\|^{2}-\sum_{k=1}^{p}\left\|\mathbb{E}_{x v_{k}}\left(\mathbb{E}_{v_{k} v_{k}}^{1 / 2}\right)^{\dagger}\right\|^{2}
$$

Proof. It follows from (55) that the unconstrained minimum (6) is achieved if $f$ is defined by (63) and if $F_{k}$ satisfies the equation $F_{k} \mathbb{E}_{v_{k} v_{k}}^{1 / 2}-\mathbb{E}_{x v_{k}}\left(\mathbb{E}_{v_{k} v_{k}}^{1 / 2}\right)^{\dagger}=\mathbb{O}$ for each $k=1, \ldots, p$. Similar to (57)-(58), its general solution is given by

$$
F_{k}=\bar{F}_{k}=\mathbb{E}_{x v_{k}} \mathbb{E}_{v_{k} v_{k}}^{\dagger}+A_{k}\left[I-\mathbb{E}_{v_{k} v_{k}} \mathbb{E}_{v_{k} v_{k}}^{\dagger}\right]
$$

because $\mathbb{E}_{v_{k} v_{k}}^{1 / 2}\left(\mathbb{E}_{v_{k} v_{k}}^{1 / 2}\right)^{\dagger}=\mathbb{E}_{v_{k} v_{k}} \mathbb{E}_{v_{k} v_{k}}^{\dagger}$. We define $\overline{\mathcal{F}}_{k}$ by $\left[\overline{\mathcal{F}}_{k}\left(\boldsymbol{w}_{k}\right)\right](\omega)=\bar{F}_{k}\left[\boldsymbol{w}_{k}(\omega)\right]$ for all $k=$ $1, \ldots, p$, and then (64)-(65) are true. The relation (66) follows from (55) and (63)-(65).

Remark 5. The difference between the transforms given by Theorems 1 and 2 is that $\mathcal{F}_{k}^{0}$ by $(20)$ (Theorem 1) does not contain a factor associated with $\left(\mathbb{E}_{v_{k} v_{k}}^{1 / 2}\right)^{\dagger}$ for all $k=1, \ldots p$. A similar observation is true for Corollaries 1 and 2 .

Remark 6. The transforms given by Theorems 1 and 2 are not unique due to arbitrary operators $\mathcal{A}_{1}, \ldots, \mathcal{A}_{p}$. A natural particular choice is $\mathcal{A}_{1}=\cdots=\mathcal{A}_{p}=\mathbb{O}$.

\subsubsection{Compression procedure by $\mathcal{T}_{p}^{0}$}

Let us consider transform $\mathcal{T}_{p}^{0}$ given by (8), (43)-(45) with $A_{k}=\mathbb{O}$ for $k=1, \ldots, p$ where $A_{k}$ is the matrix given in $(58)$. We write $\left[\mathcal{T}_{p}^{0}(\boldsymbol{y})\right](\omega)=T_{p}^{0}(y)$ with $T_{p}^{0}: \mathbb{R}^{n} \rightarrow \mathbb{R}^{m}$.

Let

$$
B_{k}^{(1)}=S_{k \eta_{k}} V_{k \eta_{k}} D_{k \eta_{k}}^{T} \quad \text { and } \quad B_{k}^{(2)}=D_{k \eta_{k}}^{T}\left(\mathbb{E}_{v_{k} v_{k}}^{1 / 2}\right)^{\dagger}
$$

so that $B_{k}^{(1)} \in \mathbb{R}^{m \times \eta_{k}}$ and $B_{k}^{(2)} \in \mathbb{R}^{\eta_{k} \times n}$. Here, $\eta_{1}, \ldots, \eta_{p}$ are determined by (7). Then

$$
T_{p}^{0}(y)=f+\sum_{k=1}^{p} B_{k}^{(1)} B_{k}^{(2)} v_{k}
$$

where $v_{k}=\boldsymbol{v}_{k}(\omega)$ and $B_{k}^{(2)} v_{k} \in \mathbb{R}^{\eta_{k}}$ for $k=1, \ldots, p$ with $\eta_{1}+\cdots+\eta_{p}<m$. Hence, matrices $B_{1}^{(2)}, \ldots, B_{p}^{(2)}$ perform compression of the data presented by $v_{1}, \ldots, v_{p}$. Matrices $B_{1}^{(1)}, \ldots, B_{p}^{(1)}$ perform reconstruction of the reference signal from the compressed data.

The compression ratio of transform $\mathcal{T}_{p}^{0}$ is given by

$$
r^{0}=\left(\eta_{1}+\cdots+\eta_{p}\right) / m .
$$




\subsubsection{A special case of transform $\mathcal{T}_{p}$}

The results above have been derived for any operators $\varphi_{1}, \ldots, \varphi_{p}$ in the model $\mathcal{T}_{p}$. Some specializations for $\varphi_{1}, \ldots, \varphi_{p}$ were given in Section 3.2. Here and in Section 5.2.5, we consider alternative forms for $\varphi_{1}, \ldots, \varphi_{p}$.

(i) Operators $\varphi_{1}, \ldots, \varphi_{p}$ can be determined by a recursive procedure given below. The motivation follows from the observation that performance of the transform $\mathcal{T}_{p}$ is improved if $\boldsymbol{y}$ in (5) is replaced by an estimate of $\boldsymbol{x}$.

First, we set $\boldsymbol{\varphi}_{k}(\boldsymbol{y})=\boldsymbol{y}$ and determine estimate $\boldsymbol{x}^{(1)}$ of $\boldsymbol{x}$ from the solution of problem (6) (with no constraints (7)) by Corollaries 1 or 2 with $p=1$. Next, we put $\boldsymbol{\varphi}_{1}(\boldsymbol{y})=\boldsymbol{y}$ and $\boldsymbol{\varphi}_{2}(\boldsymbol{y})=\boldsymbol{x}^{(1)}$, and find estimate $\boldsymbol{x}^{(2)}$ from the solution of unconstrained problem (6) with $p=2$. In general, for $j=1, \ldots, p$, we define $\boldsymbol{\varphi}_{j}(\boldsymbol{y})=\boldsymbol{x}^{(j-1)}$, where $\boldsymbol{x}^{(j-1)}$ has been determined similarly to $\boldsymbol{x}^{(2)}$ from the previous steps. In particular, $\boldsymbol{x}^{(0)}=\boldsymbol{y}$.

(ii) Operators $\boldsymbol{\varphi}_{1}, \ldots, \boldsymbol{\varphi}_{p}$ can also be chosen as elementary functions. An example is given in item (i) of Section 3.2 where $\boldsymbol{\varphi}_{k}(\boldsymbol{y})$ was constructed from the power functions. An alternative possibility is to choose trigonometric functions for constructing $\boldsymbol{\varphi}_{k}(\boldsymbol{y})$. For instance, one can put

$$
\left[\boldsymbol{\varphi}_{1}(\boldsymbol{y})\right](\omega)=y \quad \text { and } \quad\left[\boldsymbol{\varphi}_{k+1}(\boldsymbol{y})\right](\omega)=\left[\cos \left(k y_{1}\right), \ldots, \cos \left(k y_{n}\right)\right]^{T}
$$

with $y=\left[y_{1}, \ldots, y_{n}\right]^{T}$ and $k=1, \ldots, p-1$. In this paper, we do not analyse such a possible choice for $\varphi_{1}, \ldots, \varphi_{p}$.

\subsubsection{Other particular cases of transform $\mathcal{T}_{p}$ and comparison with known transforms}

(i) Optimal non-linear filtering. The transforms $\hat{\mathcal{T}}_{p}(36)-(37)$ and $\overline{\mathcal{T}}_{p}(63)-(65)$, which are particular cases of the transforms given in Theorems 1 and 2, represent optimal filters that perform pure filtering with no signal compression. Therefore they are important in their own right.

(ii) The Fourier series as a particular case of transform $\overline{\mathcal{T}}_{p}$. For the case of the minimization problem $(6)$ with no constraint $(7), \mathcal{F}_{1}, \ldots, \mathcal{F}_{p}$ are determined by the expressions (36) and (63)-(65) which are similar to those for the Fourier coefficients [35]. The structure of the model $\mathcal{T}_{p}$ presented by (3) is different, of course, from that for the Fourier series and Fourier polynomial (i.e. a truncated Fourier series) in Hilbert space [35]. The differences are that $\mathcal{T}_{p}$ transforms $\boldsymbol{y}$ (not $\boldsymbol{x}$ as the Fourier polynomial does) and that $\mathcal{T}_{p}$ consists of a combination of three operators $\varphi_{k}, \mathcal{Q}_{k}$ and $\mathcal{F}_{k}$ where $\mathcal{F}_{k}: L^{2}\left(\Omega, \tilde{H}_{k}\right) \rightarrow L^{2}\left(\Omega, H_{X}\right)$ is an operator, not a scalar as in the Fourier series [35]. The solutions (36) and (63)-(65) of the unconstrained problem (6) are given in terms of the observed vector $\boldsymbol{y}$, not in terms of the basis of $\boldsymbol{x}$ as in the Fourier series/polynomial. The special features of $\mathcal{T}_{p}$ require special computation methods as described in Section 5.

Here, we show that the Fourier series is a particular case of the transform $\mathcal{T}_{p}$.

Let $\boldsymbol{x} \in L^{2}(\Omega, H)$ with $H$ a Hilbert space, and let $\left\{\boldsymbol{v}_{1}, \boldsymbol{v}_{2}, \ldots\right\}$ be an orthonormal basis in $L^{2}(\Omega, H)$. For any $\boldsymbol{g}, \boldsymbol{h} \in L^{2}(\Omega, H)$, we define the scalar product $\langle\cdot, \cdot\rangle$ and the norm $\|\cdot\|_{E}$ in $L^{2}(\Omega, H)$ by

$$
\langle\boldsymbol{g}, \boldsymbol{h}\rangle=\int_{\Omega} \boldsymbol{g}(\omega) \boldsymbol{h}(\omega) d \mu(\omega) \quad \text { and } \quad\|\boldsymbol{g}\|_{E}=\langle\boldsymbol{g}, \boldsymbol{g}\rangle^{1 / 2},
$$

respectively. In particular, if $H=\mathbb{R}^{m}$ then

$$
\|\boldsymbol{g}\|_{E}^{2}=\int_{\Omega} \boldsymbol{g}(\omega)[\boldsymbol{g}(\omega)]^{T} d \mu(\omega)=\int_{\Omega}\|\boldsymbol{g}(\omega)\|^{2} d \mu(\omega)=E\left[\|\boldsymbol{g}\|^{2}\right],
$$

i.e. $E\left[\|\boldsymbol{g}\|^{2}\right]$ is defined similarly to that in (4). 
Let us consider the special case of transform $\mathcal{T}_{p}$ presented in item (iii) of Section 3.2 and let us also consider the unconstrained problem (6) formulated in terms of such a $\mathcal{T}_{p}$ where we now assume that $\boldsymbol{x}$ has the zero mean, $f=\mathbb{O}, p=\infty,\left\{\boldsymbol{v}_{1}, \boldsymbol{v}_{2}, \ldots\right\}$ is an orthonormal basis in $L^{2}(\Omega, H)$ and $\mathcal{F}_{k}$ is a scalar, not an operator as before. We denote $\alpha_{k}=\mathcal{F}_{k}$ with $\alpha_{k} \in \mathbb{R}$. Then similar to (36) in Corollary 1, the solution to unconstrained problem (6) is defined by $\hat{\alpha}_{k}$ such that

$$
\hat{\alpha}_{k}=\mathbb{E}_{x v_{k}} \quad \text { with } \quad k=1,2, \ldots
$$

Here, $\mathbb{E}_{x v_{k}}=E\left[\boldsymbol{x} \boldsymbol{v}_{k}\right]-E[\boldsymbol{x}] E\left[\boldsymbol{v}_{k}\right]=E\left[\boldsymbol{x} \boldsymbol{v}_{k}\right]=\left\langle\boldsymbol{x}, \boldsymbol{v}_{k}\right\rangle$ since $E[\boldsymbol{x}]=0$ by the assumption. Hence, $\hat{\alpha}_{k}=\mathbb{E}_{x v_{k}}$ is the Fourier coefficient and the considered particular case of $\mathcal{T}_{p}(\boldsymbol{y})$ with $\mathcal{F}_{k}$ determined by $\hat{\alpha}_{k}$ is given by

$$
\mathcal{T}_{p}(\boldsymbol{y})=\sum_{k=1}^{\infty}\left\langle\boldsymbol{x}, \boldsymbol{v}_{k}\right\rangle \boldsymbol{v}_{k}
$$

Thus, the Fourier series (71) in Hilbert space follows from (3), (6) and (36) when $\mathcal{T}_{p}$ has the form given in item (iii) of Section 3.2 with $\boldsymbol{x}, f, p,\left\{\boldsymbol{v}_{1}, \boldsymbol{v}_{2}, \ldots\right\}$ and $\mathcal{F}_{k}$ as above.

(iii) The Wiener filter as a particular case of transform $\overline{\mathcal{T}}_{p}(63)-(65)$. In the following Corollaries 3 and 4 , we show that the filter $\overline{\mathcal{T}}_{p}$ guarantees better accuracy than that of the Wiener filter.

Corollary 3. Let $p=1, E[\boldsymbol{x}]=0, E[\boldsymbol{y}]=0, \boldsymbol{\varphi}_{1}=\mathcal{I}, \mathcal{Q}_{1}=\mathcal{I}$ and $A_{1}=\mathbb{O}$ or $A_{1}=E_{x y} E_{y y}^{\dagger}$. Then $\overline{\mathcal{T}}_{p}$ is reduced to the filter $\check{\mathcal{T}}$ such that

$$
[\check{\mathcal{T}}(\boldsymbol{y})](\omega)=\check{T}[\boldsymbol{y}(\omega)]
$$

with

$$
\check{T}=E_{x y} E_{y y}^{\dagger} .
$$

Remark 7. The unconstrained linear filter, given by (72), has been proposed in [7]. The filter (72) is treated as a generalisation of the Wiener filter.

Let $\tilde{\boldsymbol{x}}, \tilde{\boldsymbol{v}}_{1}, \ldots, \tilde{\boldsymbol{v}}_{p}$ be the zero mean vectors. The transform $\overline{\mathcal{T}}_{p}$, applied to $\tilde{\boldsymbol{x}}, \tilde{\boldsymbol{v}}_{1}, \ldots, \tilde{\boldsymbol{v}}_{p}$, is denoted by $\overline{\mathcal{T}}_{W, p}$.

Corollary 4. The error $E\left[\left\|\tilde{\boldsymbol{x}}-\overline{\mathcal{T}}_{W, p}(\tilde{\boldsymbol{y}})\right\|^{2}\right]$ associated with the transform $\overline{\mathcal{T}}_{W, p}$ is smaller than the error $E\left[\|\tilde{\boldsymbol{x}}-\check{\mathcal{T}}(\tilde{\boldsymbol{y}})\|^{2}\right]$ associated with the Wiener filter [7] by $\sum_{k=2}^{p}\left\|E_{\tilde{x} \tilde{v}_{k}}\left(E_{\tilde{v}_{k} \tilde{v}_{k}}^{1 / 2}\right)^{\dagger}\right\|^{2}$, i.e.

$$
E\left[\left\|\tilde{\boldsymbol{x}}-\overline{\mathcal{T}}_{W, p}(\tilde{\boldsymbol{y}})\right\|^{2}\right]=E\left[\|\tilde{\boldsymbol{x}}-\check{\mathcal{T}}(\tilde{\boldsymbol{y}})\|^{2}\right]-\sum_{k=2}^{p}\left\|E_{\tilde{x} \tilde{v}_{k}}\left(E_{\tilde{v}_{k} \tilde{v}_{k}}^{1 / 2}\right)^{\dagger}\right\|^{2} .
$$

Proof. It is easy to show that

$$
E\left[\|\tilde{\boldsymbol{x}}-\check{\mathcal{T}}(\tilde{\boldsymbol{y}})\|^{2}\right]=\left\|E_{\tilde{x} \tilde{x}}^{1 / 2}\right\|^{2}-\left\|E_{\tilde{x} \tilde{v}_{1}}\left(E_{\tilde{v_{1}} \tilde{v}_{1}}^{1 / 2}\right)^{\dagger}\right\|^{2},
$$

and then (73) follows from (66) and (74).

(iv) The KLT as a particular case of transform $\mathcal{T}_{p}^{0}$ (43)-(46). The KLT [7] follows from (43)-(46) as a particular case if $f=\mathbb{O}, p=1, \boldsymbol{\varphi}_{1}=\mathcal{I}, \mathcal{Q}_{1}=\mathcal{I}$ and $A_{1}=\mathbb{O}$.

To compare the transform $\mathcal{T}_{p}^{0}$ with the KLT [7], we apply $\mathcal{T}_{p}^{0}$, represented by (43)-(46), to the zero mean vectors $\tilde{\boldsymbol{x}}, \tilde{\boldsymbol{v}}_{1}, \ldots, \tilde{\boldsymbol{v}}_{p}$ as above. We write $\mathcal{T}_{p}^{*}$ for such a version of $\mathcal{T}_{p}^{0}$, and $\mathcal{T}_{\text {KLT }}$ for the KLT [7]. 
Corollary 5. The error $E\left[\left\|\tilde{\boldsymbol{x}}-\mathcal{T}_{p}^{*}(\tilde{\boldsymbol{y}})\right\|^{2}\right]$ associated with the transform $\mathcal{T}_{p}^{*}$ is smaller than the error $E\left[\left\|\tilde{\boldsymbol{x}}-\mathcal{T}_{\mathrm{KLT}}(\tilde{\boldsymbol{y}})\right\|^{2}\right]$ associated with the KLT [7] by $\sum_{k=2}^{p} \sum_{j=1}^{\eta_{k}} \beta_{k j}^{2}$, i.e.

$$
E\left[\left\|\tilde{\boldsymbol{x}}-\mathcal{T}_{p}^{*}(\tilde{\boldsymbol{y}})\right\|^{2}\right]=E\left[\left\|\tilde{\boldsymbol{x}}-\mathcal{T}_{\mathrm{KLT}}(\tilde{\boldsymbol{y}})\right\|^{2}\right]-\sum_{k=2}^{p} \sum_{j=1}^{\eta_{k}} \beta_{k j}^{2} .
$$

Proof. The error associated with $\mathcal{F}_{\mathrm{KLT}}[7]$ is represented by (46) for $p=1$,

$$
E\left[\left\|\tilde{\boldsymbol{x}}-\mathcal{T}_{\mathrm{KLT}}(\tilde{\boldsymbol{y}})\right\|^{2}\right]=\left\|E_{\tilde{x} \tilde{x}}^{1 / 2}\right\|^{2}-\sum_{j=1}^{\eta_{1}} \beta_{1 j}^{2} .
$$

Then (75) follows from (46) and (76).

(v) The transform [26] as a particular case of transform $\mathcal{T}_{p}^{0}$. The transform [26] follows from (3) as a particular case if $f=\mathbb{O}, p=2, \boldsymbol{\varphi}_{1}(\boldsymbol{y})=\boldsymbol{y}, \boldsymbol{\varphi}_{2}(\boldsymbol{y})=\boldsymbol{y}^{2}$ and $\mathcal{Q}_{1}=\mathcal{Q}_{2}=\mathcal{I}$ where $\boldsymbol{y}^{2}$ is defined by $\boldsymbol{y}^{2}(\omega)=\left[y_{1}^{2}, \ldots, y_{n}^{2}\right]^{T}$. We note that transform [26] has been generalized in [27].

(vi) The transforms [27] as particular cases of transform $\mathcal{T}_{p}$. The transform [27] follows from (3) if $\mathcal{Q}_{k}=\mathcal{I}, \boldsymbol{\varphi}_{k}(\boldsymbol{y})=\boldsymbol{y}^{k}$ where $\boldsymbol{y}^{k}=(\boldsymbol{y}, \ldots, \boldsymbol{y}) \in L^{2}\left(\Omega, \mathbb{R}^{n k}\right), \mathbb{R}^{n k}$ is the $k$ th degree of $\mathbb{R}^{n}$, and if $\mathcal{F}_{k}$ is a $k$-linear operator.

To compare transform $\mathcal{T}_{p}^{0}$ and transform $\mathcal{T}_{[27]}$ [27] of rank $r$, we write $z_{j}=y_{j} y, z=$ $\left[z_{1}, \ldots, z_{n}\right]^{T}, s=\left[\begin{array}{lll}1 & y^{T} & z^{T}\end{array}\right]^{T}$ and denote by $\alpha_{1}, \ldots, \alpha_{r}$ the non-zero singular values associated with the truncated SVD for the matrix $\mathbb{E}_{x s}\left(\mathbb{E}_{s s}^{1 / 2}\right)^{\dagger}$. Such a SVD is constructed similarly to that in $(39)-(41)$.

Corollary 6. Let $\Delta_{p}=\sum_{k=1}^{p} \sum_{j=1}^{\eta_{k}} \beta_{k j}^{2}-\sum_{j=1}^{r} \alpha_{j}^{2}$ and let $\Delta_{p} \geq 0$. The error $E\left[\left\|\boldsymbol{x}-\mathcal{T}_{p}^{0}(\boldsymbol{y})\right\|^{2}\right]$ associated with the transform $\mathcal{T}_{p}^{0}$ is less than the error $E\left[\left\|\boldsymbol{x}-\mathcal{T}_{[27]}(\boldsymbol{y})\right\|^{2}\right]$ associated with the transform $\mathcal{T}_{[27]}$ by $\Delta_{p}$, i.e.

$$
E\left[\left\|\boldsymbol{x}-\mathcal{T}_{p}^{0}(\boldsymbol{y})\right\|^{2}\right]=E\left[\left\|\boldsymbol{x}-\mathcal{T}_{[27]}(\boldsymbol{y})\right\|^{2}\right]-\Delta_{p} .
$$

Proof. It follows from [27] that

$$
E\left[\left\|\boldsymbol{x}-\mathcal{T}_{[27]}(\boldsymbol{y})\right\|^{2}\right]=\left\|E_{x x}^{1 / 2}\right\|^{2}-\sum_{j=1}^{r} \alpha_{j}^{2} .
$$

Then (77) follows from (46) and (78).

We note that, in general, a theoretical verification of the condition $\Delta_{p} \geq 0$ is not straightforward. At the same time, for any particular $\boldsymbol{x}$ and $\boldsymbol{y}, \Delta_{p}$ can be estimated numerically.

Although the transform $\mathcal{T}_{p}^{0}$ includes the transform $\mathcal{T}_{[27]}$, the accuracy of $\mathcal{T}_{[27]}$ is, in general, better than that of $\mathcal{T}_{p}^{0}$ for the same degrees of $\mathcal{T}_{p}^{0}$ and $\mathcal{T}_{[27]}$. This is because $\mathcal{T}_{[27]}$ implies more terms. For instance, $\mathcal{T}_{[27]}$ of degree two consists of $n+1$ terms while $\mathcal{T}_{p}^{0}$, for $p=2$, consists of three terms only. If for a given $p$, the condition $\Delta_{p} \geq 0$ is not fulfilled, then the accuracy $E\left[\left\|\boldsymbol{x}-\mathcal{T}_{p}^{0}(\boldsymbol{y})\right\|^{2}\right]$ can be improved by increasing $p$ or by applying the iterative method presented in $[28]$.

(vii) Unlike the techniques presented in [13, 14], our method implements simultaneous filtering and compression, and provides this data processing in probabilistic setting. The idea of implicitly mapping the data into a high-dimensional feature space $[8,12,15]$ could be extended to the transform presented in this paper. We intend to develop such an extension in the future. 


\section{Numerical realization}

6.1. Orthogonalization. Numerical realization of transforms of random vectors implies a representation of observed data and estimates of covariance matrices in the form of associated samples.

For the random vector $\boldsymbol{u}_{k}$, we have $q$ realizations, which are concatenated into $n \times q$ matrix $U_{k}$. A column of $U_{k}$ is a realization of $\boldsymbol{u}_{k}$. Thus, a sequence of vectors $\boldsymbol{u}_{1}, \ldots, \boldsymbol{u}_{p}$ is represented by a sequence of matrices $U_{1}, \ldots, U_{p}$. Therefore the transformation of $\boldsymbol{u}_{1}, \ldots, \boldsymbol{u}_{p}$ to orthonormal or orthogonal vectors $\boldsymbol{v}_{1}, \ldots, \boldsymbol{v}_{p}$ (by Lemmata 1 and 3) is reduced to a procedure for matrices $U_{1}, \ldots, U_{p}$ and $V_{1}, \ldots, V_{p}$. Here, $V_{k} \in \mathbb{R}^{n \times q}$ is a matrix formed from realizations of the random vector $\boldsymbol{v}_{k}$ for each $k=1, \ldots, p$.

Alternatively, matrices $V_{1}, \ldots, V_{p}$ can be determined from known procedures for matrix orthogonalization [43]. In particular, the QR decomposition [43] can be exploited in the following way. Let us form a matrix $U=\left[U_{1}^{T} \ldots U_{p}^{T}\right]^{T} \in \mathbb{R}^{n p \times q}$ where $p$ and $q$ are chosen such that $n p=q$, i.e. $U$ is square ${ }^{3}$. Let

$$
U=V R
$$

be the $\mathrm{QR}$ decomposition for $U$ with $V \in \mathbb{R}^{n p \times q}$ orthogonal and $R \in \mathbb{R}^{n p \times q}$ upper triangular. Next, we write $V=\left[V_{1}^{T} \ldots V_{p}^{T}\right]^{T} \in \mathbb{R}^{m p \times q}$ where $V_{k} \in \mathbb{R}^{n \times q}$ for $k=1, \ldots, p$. The submatrices $V_{1}, \ldots, V_{p}$ of $V$ are orthogonal, i.e. $V_{i} V_{j}^{T}=\left\{\begin{array}{ll}\mathbb{O}, & i \neq j, \\ I, & i=j,\end{array}\right.$ for $i, j=1, \ldots, p$, as required.

Other known procedures for matrix orthogonalization can be applied to $U_{1}, \ldots, U_{p}$ in a similar fashion.

Remark 8. For the cases when $\boldsymbol{v}_{1}, \ldots, \boldsymbol{v}_{p}$ are orthonormal or orthogonal but not orthonormal, the associated accuracies $(21),(38),(46)$ and (66) differ for the factors depending on $\left(\mathbb{E}_{v_{k} v_{k}}^{1 / 2}\right)^{\dagger}$. In the case of orthonormal $\boldsymbol{v}_{1}, \ldots, \boldsymbol{v}_{p},\left(\mathbb{E}_{v_{k} v_{k}}^{1 / 2}\right)^{\dagger}=I$ and this circumstance can lead to an increase in the accuracy.

6.2. Covariance matrices. The expectations and covariance matrices in Lemmata 1-3 and Theorems 1-2 can be estimated, for example, by the techniques developed in [36, 37, 38, $39,40,41]$. We note that such estimation procedures represent specific problems which are not considered here.

6.3. $\mathcal{T}_{p}^{0}, \hat{\mathcal{T}}_{p}$ and $\overline{\mathcal{T}}_{p}$ for zero mean vectors. The computational work for $\mathcal{T}_{p}^{0}$ (Theorems 1 and 2), $\hat{\mathcal{T}}_{p}$ and $\overline{\mathcal{T}}_{p}$ (Corollaries 1 and 2) can be reduced if $\mathcal{T}_{p}^{0}, \hat{\mathcal{T}}_{p}$ and $\overline{\mathcal{T}}_{p}$ are applied to the zero mean vectors $\tilde{\boldsymbol{x}}, \tilde{\boldsymbol{v}}_{1}, \ldots, \tilde{\boldsymbol{v}}_{p}$ given by $\tilde{\boldsymbol{x}}=\boldsymbol{x}-E[\boldsymbol{x}], \tilde{\boldsymbol{v}}_{1}=\boldsymbol{v}_{1}-E\left[\boldsymbol{v}_{1}\right], \ldots, \tilde{\boldsymbol{v}}_{p}=\boldsymbol{v}_{p}-E\left[\boldsymbol{v}_{p}\right]$. Then $f^{0}=\mathbb{O}$ and $\bar{f}=\mathbb{O}$. The estimates of the original $\boldsymbol{x}$ are then given by

$$
\check{\boldsymbol{x}}=E[\boldsymbol{x}]+\sum_{k=1}^{p} \mathcal{F}_{k}^{0}\left(\tilde{\boldsymbol{v}}_{k}\right), \quad \hat{\boldsymbol{x}}=E[\boldsymbol{x}]+\sum_{k=1}^{p} \hat{\mathcal{F}}_{k}\left(\tilde{\boldsymbol{v}}_{k}\right) \quad \text { and } \quad \quad \overline{\boldsymbol{x}}=E[\boldsymbol{x}]+\sum_{k=1}^{p} \overline{\mathcal{F}}_{k}\left(\tilde{\boldsymbol{v}}_{k}\right)
$$

respectively. Here, $\mathcal{F}_{k}^{0}, \hat{\mathcal{F}}_{k}$ and $\overline{\mathcal{F}}_{k}$ are defined similarly to (20), (36), (44), (45), (64) and (65).

\section{Discussion}

Some distinctive features of the proposed techniques are summarized as follows.

Remark 9. It follows from Theorems 1 and 2, and Corollaries 1 and 2 that the accuracy associated with the proposed transform improvs when $p$ increases.

\footnotetext{
${ }^{3}$ Matrix $U$ can also be presented as $U=\left[U_{1} \ldots U_{p}\right]$ with $p$ and $q$ such that $n=p q$.
} 
Remark 10. Unlike the approaches based on Volterra polynomials [27, 29, 34] our method does not require computation of pseudo-inverses for large $N \times N$ matrices with $N=n+n^{2}+\cdots+n^{p-1}$. Instead, the proposed transforms use pseudo-inverses of $n \times n$ matrix $\mathbb{E}_{v_{k} v_{k}}$. See Theorems 1 and 2. This leads to a substantial reduction in computational work.

Remark 11. The idea of the recurrent transform [28] can be extended for the proposed transform in a way similar to that considered in [28]. The authors intend to develop a theory for such an extension in a feasible future.

\section{Conclusions}

The new results obtained in the paper are summarized as follows.

We have proposed a new approach to constructing optimal nonlinear transforms for random vectors. The approach is based on a representation of a transform in the form of the sum of $p$ reduced-rank transforms. Each particular transform is formed by the linear reduced-rank operator $\mathcal{F}_{k}$, and by operators $\varphi_{k}$ and $\mathcal{Q}_{k}$ with $k=1, \ldots, p$. Such a device allows us to improve the numerical characteristics (accuracy, compression ration and computational work) of the known transforms based on the Volterra polynomial structure [27, 29, 34]. These objectives are achieved due to the special "intermediate" operators $\varphi_{1}, \ldots, \varphi_{p}$ and $\mathcal{Q}_{1}, \ldots, \mathcal{Q}_{p}$. In particular, we have proposed two types of orthogonalizing operators $\mathcal{Q}_{1}, \ldots, \mathcal{Q}_{p}$ (Lemmata 1 and 3) and a specific method for determining $\varphi_{1}, \ldots, \boldsymbol{\varphi}_{p}$ (Section 5.2.4). Such operators reduce the determination of optimal linear reduced-rank operators $\mathcal{F}_{1}^{0}, \ldots, \mathcal{F}_{p}^{0}$ to the computation of a sequence of relatively small matrices (Theorems 1 and 2).

Particular cases of the proposed transform, which follow from the solution of the unconstrained minimization problem (6), have been presented in Corollaries 1 and 2. Such transforms are treated as new optimal nonlinear filters and, therefore, are important in their own right.

The explicit representations of the accuracy associated with the proposed transforms have been rigorously justified in Theorems 1 and 2, and Corollaries 1 and 2 .

It has been shown that the proposed approach generalizes the Fourier series in Hilbert space (Section 5.2.5), the Wiener filter, the Karhunen-Loève transform (KLT) and the known optimal transforms $[26,27,29]$. See Corollaries 3, 4 and 5, and Section 5.2.5 in this regard. In particular, it has been shown that the accuracies associated with the proposed transforms are better than those of the Wiener filter (Corollary 4) and the KLT (Corollary 5).

\section{A Appendix}

Proof of Lemma 1. Let us write

$$
\boldsymbol{w}_{1}=\boldsymbol{u}_{1} \quad \text { and } \quad \boldsymbol{w}_{i}=\boldsymbol{u}_{i}-\sum_{k=1}^{i-1} \mathcal{U}_{i k}\left(\boldsymbol{w}_{k}\right) \quad \text { for } \quad i=1, \ldots, p,
$$

with $\mathcal{U}_{i k}: L^{2}\left(\Omega, \mathbb{R}^{n}\right) \rightarrow L^{2}\left(\Omega, \mathbb{R}^{n}\right)$ chosen so that, for $k=1, \ldots, i-1$,

$$
\mathbb{E}_{w_{i} w_{k}}=\mathbb{O} \quad \text { if } i \neq k \text {. }
$$

We wish $(79)$ is true for any $k$, i.e.

$$
\begin{aligned}
\mathbb{E}_{w_{i} w_{k}} & =E_{w_{i} w_{k}}-E\left[\boldsymbol{w}_{i}\right] E\left[\boldsymbol{w}_{k}^{T}\right] \\
& =E\left[\left(\boldsymbol{u}_{i}-\sum_{l=1}^{i-1} \mathcal{U}_{i l}\left(\boldsymbol{w}_{l}\right)\right) \boldsymbol{w}_{k}^{T}\right]-E\left[\left(\boldsymbol{u}_{i}-\sum_{l=1}^{i-1} \mathcal{U}_{i l}\left(\boldsymbol{w}_{l}\right)\right)\right] E\left[\boldsymbol{w}_{k}^{T}\right]
\end{aligned}
$$




$$
\begin{aligned}
& =E_{u_{i} w_{k}}-U_{i k} E_{w_{k} w_{k}}-E\left[\boldsymbol{u}_{i}\right] E\left[\boldsymbol{w}_{k}^{T}\right]+E\left[\boldsymbol{w}_{k}\right] E\left[\boldsymbol{w}_{k}^{T}\right] \\
& =\mathbb{E}_{u_{i} w_{k}}-U_{i k} \mathbb{E}_{w_{k} w_{k}}=\mathbb{O} .
\end{aligned}
$$

Thus, $U_{i k}=\mathbb{E}_{u_{i} w_{k}} \mathbb{E}_{w_{k} w_{k}}^{-1}$, and the statement (i) is true.

It is clear that vectors $\boldsymbol{v}_{1}, \ldots, \boldsymbol{v}_{p}$, defined by (12), are orthogonal. For $\mathcal{Q}_{k}$, defined by (13), we have $Q_{k}=\left(\mathcal{E}_{w_{k} w_{k}}^{1 / 2}\right)^{-1}$ and

$$
\begin{aligned}
\mathbb{E}_{v_{k} v_{k}} & =E\left[\left(\mathbb{E}_{w_{k} w_{k}}^{1 / 2}\right)^{-1} \boldsymbol{w}_{k} \boldsymbol{w}_{k}^{T}\left(\mathbb{E}_{w_{k} w_{k}}^{1 / 2}\right)^{-1}\right]-E\left[\left(\mathbb{E}_{w_{k} w_{k}}^{1 / 2}\right)^{-1} \boldsymbol{w}_{k}\right] E\left[\boldsymbol{w}_{k}^{T}\left(\mathbb{E}_{w_{k} w_{k}}^{1 / 2}\right)^{-1}\right] \\
& =\left(\mathbb{E}_{w_{k} w_{k}}^{1 / 2}\right)^{-1} \mathbb{E}_{w_{k} w_{k}}\left(\mathbb{E}_{w_{k} w_{k}}^{1 / 2}\right)^{-1}=I
\end{aligned}
$$

Hence, $\boldsymbol{v}_{1}, \ldots, \boldsymbol{v}_{p}$, defined by (12), are orthonormal.

Proof of Lemma 3. We wish that $\mathbb{E}_{v_{i} v_{k}}=\mathbb{O}$ for $i \neq k$. If $Z_{i k}$ has been chosen so that this condition is true for all $k=1, \ldots, i-1$ then we have

$$
E\left[\left(\boldsymbol{u}_{i}-\sum_{l=1}^{i-1} \mathcal{Z}_{i l}\left(\boldsymbol{v}_{l}\right)\right) \boldsymbol{v}_{k}^{T}\right]=\mathbb{E}_{u_{i} v_{k}}-\sum_{l=1}^{i-1} Z_{i l} \mathbb{E}_{v_{l} v_{k}}=\mathbb{E}_{u_{i} v_{k}}-Z_{i k} \mathbb{E}_{v_{k} v_{k}}=\mathbb{O}
$$

Thus,

$$
Z_{i k} \mathbb{E}_{v_{k} v_{k}}=\mathbb{E}_{u_{i} v_{k}} .
$$

The necessary and sufficient condition [45] for the solution of the matrix equation (81) is given by

$$
\mathbb{E}_{u_{i} v_{k}} \mathbb{E}_{v_{k} v_{k}}^{\dagger} \mathbb{E}_{v_{k} v_{k}}=\mathbb{E}_{u_{i} v_{k}}
$$

By Lemma 2, (82) is true. Then, on the basis of [45], the general solution to (81) is given by (16).

\section{Acknowledgements}

The first co-author is grateful to Oliver Cappé for useful discussions related to the structure of the proposed transform.

[1] Hotelling H., Analysis of a complex of statistical variables into Principal Components, J. Educ. Psychol., 1933, V.24, 417-441, 498-520.

[2] Karhunen K., Über Lineare Methoden in der Wahrscheinlichkeitsrechnung, Ann. Acad. Sci. Fennicae, Ser. A, 1947, V.137.

[3] Loève M., Fonctions aléatoires de second order, in P. Lévy, Processus Stochastiques et Mouvement Brownien, Paris, Hermann, 1948.

[4] Jolliffe I.T., Principal component analysis, New York, Springer Verlag, 1986 (2 ed., 2002).

[5] Scharf L.L., The SVD and reduced rank signal processing, Signal Processing, 1991, V.25, $113-133$.

[6] Yamashita Y., Ogawa H., Relative Karhunen-Loéve transform, IEEE Trans. on Signal Processing, 1996, V.44, 371-378.

[7] Hua Y., Liu W.Q., Generalized Karhunen-Loève transform, IEEE Signal Processing Letters, 1998, V.5, $141-143$.

[8] Vapnik V., Statistical Learning Theory, Wiley, 1998.

[9] Ocaña F.A., Aguilera A.M., Valderrama M.J., Functional principal componenets analysis by choice of norm, J. Multivariate Anal., 1999, V.71, 262-276.

[10] Tipping M.E., Bishop C.M., Probabilistic principal component analysis, J. of the Royal Statistical Society, Ser. A, 1999, V.61, 611-619.

[11] Tipping M.E., Bishop C.M., Mixtures of probabilistic principal component analysers, Neural Computation, 1999, V.11, 443-482. 
[12] Schölkopf B., Smola A.J., Müller K.-R., Kernel principal component analysis, in Advances in Kernel Methods. Support Vector Learning, Editors B. Schölkopf, C.J.C. Burges and A.J. Smola, Cambridge, MIT Press, 1999, 327-352.

[13] Tenenbaum J.B., de Silva V., Langford J.C., A global geometric framework for nonlinear dimensionality reduction, Science, 2000, V.290, Issue 5500, 2319-2323.

[14] Rowers S.T., Saul L.K., Nonlinear dimensionality reduction by locally linear embedding, Science, 2000, V.290, Issue 5500, 2323-2326.

[15] Cristianini N., Shawe-Taylor J., An introduction to support vector machines and other kernel-based learning methods, Cambridge, Cambridge University Press, 2000.

[16] Yamada I., Sekiguchi T., Sakaniwa K., Reduced rank Volterra filter for robust identification of nonlinear systems, in Proc. 2nd Int. Workshop on Multidimensional (ND) Systems - NDS2000, Poland, Czocha Castle, 2000, 171-175.

[17] Hua Y., Nikpour M., Stoica P., Optimal reduced-rank estimation and filtering, IEEE Trans. on Signal Processing, 2001, V.49, 457-469.

[18] Kneip A., Utikal K.J., Inference for density families using functional principal component analysis, Journal of the American Statistical Association, 2001, V.96, 519-542.

[19] Honig M.L., Xiao W., Performance of reduced-rank linear interferrence suppression, IEEE Trans. on Information Theory, 2001, V.47, 1928-1946.

[20] Chen W., Mitra U., Schniter P., On the equivalence of three rediced rank linear estimators with applications to DS-CDMA, IEEE Trans. on Information Theory, 2002, V.48, 2609-2614.

[21] Honig M.L., Goldstein J.S., Adaptive reduced-rank interference suppression based on multistage Wiener filter, IEEE Trans. on Communications, 2002, V.50, 986-994.

[22] Stock J.H., Watson M.W., Forecasting using principal components from a large number of predictors, Journal of the American Statistical Association, 2002, V.97, 1167-1179.

[23] Fukunaga K., Introduction to statistical pattern recognition, Boston, Academic Press, 1990.

[24] Kraut S., Anderson R.H., Krolik J.L., A generalized Karhunen-Loève basis for efficient estimation of tropospheric refractivity using radar clutter, IEEE Trans. on Signal Processing, 2004, V.52, 48-60.

[25] Torokhti A., Howlett P., An optimal filter of the second order, IEEE Trans. on Signal Processing, 2001, V.49, 1044-1048.

[26] Torokhti A., Howlett P., Optimal fixed rank transform of the second degree, IEEE Trans. on Circuits and Systems. Part II, Analog \& Digital Signal Processing, 2001, V.48, 309-315.

[27] Torokhti A., Howlett P., Pearce C., New perspectives on optimal transforms of random vectors, Optimization: Theory and Applications, to appear.

[28] Torokhti A., Howlett P., Constructing fixed rank optimal estimators with method of recurrent best approximations, J. Multivariate Analysis, 2002, V.86, 293-309.

[29] Torokhti A., Howlett P., Best operator approximation in modelling of nonlinear Systems, IEEE Trans. on Circuits and Systems. Part I, Fundamental Theory and Applications, 2002, V.49, 1792-1798.

[30] Torokhti A., Howlett P., Method of recurrent best estimators of second degree for optimal filtering of random signals, Signal Processing, 2003, V.83, 1013-1024.

[31] Torokhti A., Howlett P., Best causal mathematical models for a nonlinear system, IEEE Trans. on Circuits and Systems. Part I, Fundamental Theory and Applications, to appear.

[32] Sontag E.D., Polynomial response maps, Lecture Notes in Control and Information Sciences, 1979. Vol. 13.

[33] Chen S., Billings S.A., Representation of non-linear systems: NARMAX model, Int. J. Control, 1989, V.49, 1013-1032.

[34] Howlett P.G., Torokhti A.P., Pearce C.E.M., A philosophy for the modelling of realistic non-linear systems, Proc. of Amer. Math. Soc., 2003, V.132, 353-363.

[35] Cotlar M., Cignoli R., An introduction to functional analysis, Amsterdam - London, North-Holland Publishing Company, 1974, 114-116.

[36] Perlovsky L.I., Marzetta T.L., Estimating a covariance matrix from incomplete realizations of a random vector, IEEE Trans. on Signal Processing, 1992, V.40, 2097-2100.

[37] Kauermann G., Carroll R.J., A note on the efficiency of Sandwich covariance matrix estimation, Journal of the American Statistical Association, 2001, V.96, 1387-1396.

[38] Schneider M.K., Willsky A.S., A Krylov subspace method for covariance approximation and simulation of a random process and fields, Int. J. Multidim. Syst. \& Signal Processing, 2003, V.14, 295-318. 
[39] Kubokawa T., Srivastava M.S., Estimating the covariance matrix: a new approach, J. Multivariate Analysis, 2003, V.86, 28-47.

[40] Ledoit O., Wolf M., A well-conditioned estimator for large-dimensional covariance matrices, J. Multivariate Analysis, 2004, V.88, 365-411.

[41] Leung P.L., Ng F.Y., Improved estimation of a covariance matrix in an elliptically contoured matrix distribution, J. Multivariate Analysis, 2004, V.88, 131-137.

[42] Higham N.J., Stable iterations for the matrix square root, Numerical Algorithms, 1997, V.15, $227-241$.

[43] Golub G.H., van Loan C.F., Matrix computations, Baltimore, Johns Hopkins University Press, 1996.

[44] Kowalski M.A., Sikorski K.A., Stenger F., Selected topics in approximation and computations, New York Oxford, Oxford University Press, 1995.

[45] Ben-Israel A., Greville T.N.E., Generalized inverses: theory and applications, New York, John Wiley \& Sons, 1974.

[46] Mathews V.J., Sicuranza G.L., Polynomial signal processing, J. Wiley \& Sons, 2001.

[47] Goldstein J.S., Reed I., Scharf L.L., A multistage representation of the Wiener filter based on orthogonal projections, IEEE Trans. on Information Theory, 1998, V.44, 2943-2959. 